\title{
Balancing selection and the crossing of fitness valleys in structured populations: diversification in the gametophytic self-incompatibility system
}

${ }^{1}$ Univ. Lille, CNRS, UMR 8198 - Evo-Eco-Paleo, F-59000 Lille, France, ${ }^{2}$ International Research Laboratory 3614, CNRS, 29680 Roscoff, France, ${ }^{3}$ Station Biologique de Roscoff, Sorbonne Université, Roscoff 29680, France

*corresponding author: sylvain.billiard@univ-lille.fr

10 Abstract: The self-incompatibility locus ( $S$-locus) of flowering plants displays a striking allelic diversity. How such a diversity has emerged remains unclear. In this paper, we performed numerical simulations in a finite island population genetics model to investigate how population subdivision affects the diversification process at a $S$-locus, given that the two-genes architecture typical of $S$-loci involves the crossing of a fitness valley. We show that population structure increases the number of self-incompatibility haplotypes ( $S$-haplotypes) maintained in the whole metapopulation, but at the same time also slightly reduces the parameter range allowing for their diversification. This increase is partly due to a reinforcement of the diversification and replacement dynamics of $S$-haplotypes within and among demes. We also show that the two-genes architecture leads to a higher diversity compared with a simpler genetic architecture where new $S$-haplotypes appear in a single mutation step. We conclude that population structure helps explain the large allelic diversity at the self-incompatibility locus. Overall, our results suggest that population subdivision can act in two opposite directions: it makes easier $S$-haplotypes diversification but increases the risk that the SI system is lost.

Keywords: fitness landscape, negative frequency dependent selection, metapopulation, genetic architecture, two genes epistasis 


\section{Introduction}

Adaptation often occurs on rugged fitness landscapes with multiple peaks and valleys (e.g. Korona et al. 1994; Burch and Chao 2000; Weinreich et al. 2006; Kvitek and Sherlock 2011; Salverda et al. 2011; Vos et al. 2015, for a review see de Visser and Krug 2014). This is especially true when the trait is genetically complex and the emergence of evolutionary novelties is conditioned on the beneficial combination of epistatic mutations in a single genome (Whitlock et al., 1995). In many cases, attaining a higher fitness peak can require a succession of mutations where some are deleterious on their own (Wright, 1932). In other words, adaptation can involve the crossing of fitness valleys.

The rate at which populations move across the fitness landscape and eventually cross fitness valleys depends on a subtle balance between mutation rate, population size and the distribution of mutational effects (e.g. Weissman et al. 2009; Bovier et al. 2019 in asexual panmictic populations), in combination with genetic exchange across space (e.g. Bitbol and Schwab 2014; Komarova et al. 2014; McLaren 2016 in asexual spatially divided populations), or between genomes (the recombination rate in sexual populations, e.g. Weissman et al. 2010). Overall, the rate at which fitness valleys are crossed depends on the time taken for the epistatic mutations to occur in the same genome, and the probability of fixation and time of residence of mutations in the population.

Theoretical studies investigating crossing fitness valleys have mostly assumed directional selection, so their predictions cannot be applied to all traits. Many complex genetic systems, in particular, evolve under balancing selection, including in particular pathogens escaping an immune system (Bowen and Walker, 2005; Fernandez et al., 2005; Shih et al., 2007), or the genes involved in the avoidance of self-fertilization in fungi, plants or ciliates (Billiard et al., 2011). Studying how fitness valleys are crossed in such systems is particularly relevant because they are often characterized by a very large allelic diversity at superloci, suggesting a high rate of emergence of evolutionary novelties and possibly frequent fitness valley crossing.

The self-incompatibility (SI) system in Angiosperms is particularly relevant to address this question. SI is present in about $40 \%$ of Angiosperms families, where it allows recognition between pollen and pistils for selfing avoidance (Igic et al., 2008; McCubbin and Kao, 2000; Barrett, 2002). SI is often determined by a single locus, called the $S$-locus. The $S$-locus is characterized by high haplotypic diversity maintained by a particular type of balancing selection, negative frequency-dependent selection, whereby in- 
dividuals carrying rare haplotypes can mate with more individuals than the ones carrying frequent haplotypes (Wright, 1939). Several theoretical works studied the conditions of emergence of new $S$-haplotypes under a variety of assumptions (Uyenoyama et al., 2001; Gervais et al., 2011; Sakai, 2016; Bod'ová et al., 2018; Harkness et al., 2019), in particular through an evolutionary step implying the crossing of a fitness valley.

In this study we focused on gametophytic SI (GSI), in which the pollen specificity is determined by its own haploid genome and $S$-alleles are codominant in pistils. The genetic architecture of the $S$-locus is typically relatively simple, with male and female specificities being encoded by distinct but tightly linked genes (often one each, Fujii et al., 2016, but see non-self recognition systems, e.g. Bod'ová et al., 2018). Uyenoyama et al. (2001) studied the conditions of emergence of a new $S$-haplotype by mutations on both genes. They considered that new $S$-haplotypes are formed first by a mutation on the male or female determinant, creating an intermediate haplotype with a new pollen or pistil specificity but no matching recognition in the other gene. This haplotype is then self-compatible (SC) and its selfed offspring will suffer from inbreeding depression. This first mutation can then be followed by a compensatory mutation on the female or male determinant, restoring a fully functional new $S$-haplotype in which the pistil specificity matches that of the pollen. Uyenoyama et al. (2001) showed that in an infinite population, new $S$-haplotypes can emerge for high values of inbreeding depression and low to intermediate values of the rate of self-pollination. In these conditions, the intermediate SC haplotype invades when rare but does not go to fixation, allowing the second mutation to arise and create the new $S$-haplotype. However, they also showed that the ancestral $S$-haplotype was most often lost by competitive exclusion by its mutated SC haplotype. This analysis thus showed that even though new $S$-haplotypes can emerge, the new derived $S$-haplotype replaced their ancestral copies along the process, such that in the end the overall number of $S$-haplotypes remained constant. The diversification of $S$-haplotypes is thus overall precluded either because the SC intermediate haplotype does not invade or because its ancestor is lost resulting in $S$-haplotypes turnover rather than diversification (Box 1, Fig. S1). Hence, since in both situations, the emergence of a new $S$-haplotype involves the maintenance in the population of less fit haplotypes, $S$-haplotypes diversification can be considered as a fitness valley crossing situation.

Gervais et al. (2011) further analyzed Uyenoyama et al. (2001)'s model, but in a finite population with recurrent mutations. They found that $S$ haplotype diversification can occur under a relatively wide range of parameters because the residence time of SC haplotypes can be long in finite popu- 
110 lations . Gervais et al. (2011) showed that, as in the case of directional selection (e.g. Weissman et al., 2009), the rate at which new S-haplotypes emerge depends on the balance between population size, mutation rates and selection strength (inbreeding depression cost $v s$. negative frequency-dependent selection). Gervais et al. (2011) further showed that diversification is a 115 self-attenuating process, whereby the increase in the number of extant $S$ haplotypes in the population makes the emergence of novel $S$-haplotypes in turn less likely. As a result, the maximal number of $S$-haplotypes maintained at stationary state is predicted to be a little more than a dozen, almost an order of magnitude lower than the observed haplotypic diversity in natural populations (from a few dozens to $>100$ observed $S$-haplotypes (Lawrence, 2000; Castric and Vekemans, 2004)). Hence, current models fall short in explaining the entire diversity encountered at the $S$-locus in natural populations (but see Sakai, 2016).

Uyenoyama et al. (2001) and Gervais et al. (2011) suggested that population subdivision could enhance the diversification of $S$-haplotypes, which could help resolve the discrepancy between the number of $S$-haplotypes in natural populations and theoretical predictions. Wright (1939) was the first to propose that population subdivision might contribute to the large diversity of $S$-haplotypes, but did not account for the genetic architecture underlying SI. More recent works also suggest that population subdivision could in fact have contrasted effects on $S$-haplotypes diversity and diversification. Population subdivision indeed has, on the one hand, opposed effects on the maintenance of $S$-haplotypes at the global (metapopulation) or local (deme) scale Schierup (1998); Schierup et al. (2000), and, on the other hand, it facilitates the breakdown of SI (Brom et al., 2020). How population subdivision would globally affect diversification thus remains unclear.

In this paper, our aim is to evaluate the effect of population structure on the dynamics of $S$-haplotypes diversification. Our central question is whether population structure can explain the level of haplotypic diversity observed at the $S$-locus in natural populations. First, we investigated the parameter range allowing $S$-haplotypes to diversify in structured populations compared with unstructured populations. Second, we determined how the number of $S$-haplotypes maintained at steady state varies with the degree of population structure. Third, we disentangled the mechanisms underlying 145 the effect of population structure on $S$-haplotypes diversification. Finally, in order to study the more general issue of fitness valley crossing in structured populations, we investigated the interaction between population structure and the underlying genetic architecture of the $S$-locus by comparing the number of $S$-haplotypes maintained at equilibrium in the metapopulation 


\section{Models and Methods}

\section{Genetic and demographic model}

A subdivided population of diploid hermaphroditic plants is assumed with a gametophytic SI mating system. The SI phenotype, i.e. the expressed specificities in pollen and pistils, is determined by the $S$-haplotypes of the individuals. Mating can only occur between individuals expressing different pollen and pistil specificities. Pollen specificity is determined by the haploid genome of the gametophyte (the pollen grain), and the pistil specificity is codominantly determined by the diploid genome of the sporophyte (the adult plant). $S$-haplotypes are composed of two completely linked genes: the pistil-part gene, denoted $A$, determines the female specificity; the pollenpart gene, denoted $B$, determines the male specificity. We denote $S_{i}$ an haplotype expressing specificity $i$ at the pollen and pistil genes, and $A_{i}$ and $B_{i}$ the alleles present at genes $A$ and $B$, respectively. Hence, an individual 165 with genotype $S_{i} S_{j}$ (carrying haplotypes $A_{i} B_{i} / A_{j} B_{j}$ ) is self-incompatible since its individual pollen grains express either the specificity $i$ or $j$, and its pistils express both specificities $i$ and $j$.

We ran individual-based simulations of a finite island model with nonoverlapping generations. Initially, the population was composed of selfincompatible individuals with heterozygous $S$-genotypes randomly drawn among the $n$ possible $S$ haplotypes. The metapopulation was divided into $p$ equally-sized demes of $N$ individuals. Pollen disperses at rate $d_{p}$ between demes (we neglected seed dispersal). During the life cycle, events occur in the following order: 1) Mutations at genes $A$ and $B ; 2$ ) Pollen migration between demes; 3) Pollination and fertilization; 4) Demographic events: reproduction, adults death and recruitment.

\section{Mutation}

We assumed a maximum $k$ of possible specificities in the population $(1 \leq$ $i \leq k)$. In other words, we considered a $k$-allele mutation model at both the pollen and the pistil genes. Mutations are recurrently introduced into the population at the $S$-locus. Under the 2-steps mutation model (Fig. 1B), mutations occur with probability $\mu_{2}$, on the pollen gene $\left(B_{i} \rightarrow B_{j}\right)$ or on the pistil gene $\left(A_{i} \rightarrow A_{j}\right)$, independently. A mutation randomly changes a specificity $i$ into a specificity $j \neq i(1 \leq j \leq k)$, drawn from a uniform dis- 
185

tribution. In line with the notation of Uyenoyama et al. (2001), a mutation can thus give rise to four different haplotypes, depending on the ancestral haplotype and the modified gene: 1) A pollen-part mutant self-compatible (SC) haplotype, denoted $\left.S_{b} ; 2\right)$ A pistil-part mutant SC haplotype, denoted $S_{a}$; 3) An already existing $S$-haplotype, denoted $S_{n}$; 4) A new $S$-haplotype, denoted $S_{n+1}(1 \leq n+1 \leq k)$.

The pistil- and pollen-part mutant haplotypes $S_{a}$ and $S_{b}$ are self-compatible because their pollen and pistils express different specificities. Note that haplotypes $S_{a}$ and $S_{b}$ can express a specificity $n+1$ which is not already present in the population. A new $S$-haplotype $S_{n+1}$ is formed when an ancestral haplotype has undergone two mutations: a first mutation giving a SC haplotype $\left(S_{b}\right.$ or $S_{a}$ ) and a second compensatory mutation restoring the $S$-haplotype function.

In order to compare the impact of the genetic architecture underlying the $S$-haplotype on the diversification dynamics, we also considered a 1-step mutation model. In this model, haplotype $S_{i}$ mutates in a single step to $S_{j}$ at rate $\mu_{1}$ per haploid genome per generation (i.e. both alleles $A_{i}$ and $B_{i}$ simultaneously mutate to alleles $A_{j}$ and $B_{j}$ ). $j$ is randomly and uniformly chosen such that $j \neq i$ and $1 \leq j \leq k$. This 1 -step mutation model is the one classically considered in models dealing with the evolution and maintenance of GSI (e.g. Wright, 1939; Schierup, 1998; Gervais et al., 2014; Brom et al., 2020). In this model, the $S$-locus is not split into a female and a male genes and mutations do not generate self-compatible haplotypes.

\section{Pollination}

Each individual of the next generation is formed by randomly drawing a female and a male gamete. Firstly, a female is drawn in the local deme (no seed dispersion) according to its relative fitness in the population, which differs between selfed and outcrossed individuals because of inbreeding depression $\delta$. The fitness of selfed vs. outcrossed individuals is $W_{\text {self }}=1-\delta$ and $W_{\text {out }}=1$ respectively (the probability that an individual is chosen as a mother is thus weighted by its own fitness relative to all the other individuals). Once a mother is chosen, an ovule is produced by randomly choosing one of the two chromosomes of the mother, with equal probability. Secondly, a father is drawn from the same deme as the mother with probability $1-d_{p}$ or from a different deme with probability $d_{p}$. The probability for a plant to be chosen as a father is also weighted by its own fitness because of inbreeding depression ( $W_{\text {self }}=1-\delta$ if inbred, $W_{\text {out }}=1$ if outcrossed). If the pollen grain is not compatible with the pistil of the chosen mother, 
another pollen is randomly drawn until fertilization can occur (hence all chosen ovules eventually find a compatible pollen, precluding pollen limitation). In SC individuals, compatible pollen can come either from their own pollen or from other individuals and the probability that an SC individual reproduces by selfing corresponds to the ratio of its own compatible pollen over the compatible pollen present in the total pollen pool. The effective selfing rate of an individual $i$ is thus given by

$$
a_{i}=\frac{\alpha \gamma_{i i} W_{i}}{\alpha \gamma_{i i} W_{i}+(1-\alpha)\left(1-d_{p}\right) \frac{\sum^{\frac{N}{p}-1} \gamma_{i j} W_{j}}{\frac{N}{p}-1}+(1-\alpha) d_{p} \frac{\sum^{N-\frac{N}{p}} \gamma_{i j} W_{j}}{N-\frac{N}{p}}},
$$

where $\alpha$ is the proportion of self-pollen, $\gamma_{i i}$ the number of SC haplotypes carried by individual $i, \gamma_{i j}$ the number of compatible haplotype an individual $j$ has with an individual $i\left(\gamma_{i i}\right.$ and $\gamma_{i j}$ can thus be 0,1 or 2$), W_{i}$ the pollen production of individual $i$ and $W_{j}$ the pollen production of individual $j\left(W_{i}, W_{j}=W_{\text {self }}\right.$ or $W_{\text {out }}$ depending on whether individuals $i$ and $j$ are selfed or outcrossed). The numerator and first term of denominator correspond to compatible self pollen of individual $i$, the second term of the denominator corresponds to compatible pollen of other individuals from the same deme and the third term of the denominator corresponds to compatible pollen of other individuals from other demes. The outcrossing rate of an individual $i$ is thus $1-a_{i}$.

\section{Verification of the simulations}

We first verified the validity of our simulation program by comparing its outcomes with previous results from Gervais et al. (2011) and Uyenoyama et al. (2001) for the 2-steps mutation model in a panmictic population, and from Schierup (1998) for the 1-step mutation model in a subdivided population with the same parameter values. We recovered results similar to Gervais et al. (2011) and Uyenoyama et al. (2001) (Figure S2 and Figure S3) when comparing (i) the parameters range values where allelic diversification occurred in a panmictic population of size $N=5000$; (ii) the probability of diversification in 100 replicated runs; and (iii) the dynamics of SI and SC haplotypes. We also recovered results similar to Schierup (1998) when comparing the average number of haplotypes at the scale of the whole metapopulation or of a single deme ( $<3 \%$ differences, Figure S4), for identical parameters values and the same estimation method. 


\section{Diversification probability}

Before studying the $S$-haplotype diversity at stationary state itself, we needed to determine the parameter sets where diversification can occur, and how in particular dispersal affects the probability of diversification. Simulations were repeated a hundred times for each parameter set (inbreeding depression

$240 \delta$, self-pollination rate $\alpha$, pollen dispersal rate $d_{p}$, recurrent mutation rate $\mu$, number of demes $p$ and size of the demes $N$ ). Simulations were started with $n=5$ functional $S$-alleles and with no self-compatible haplotypes. The number of possible haplotypes was set to $k=20$. Simulations were run until one of the three following events occurred (note that since haplotypes

245 could appear by mutation and be lost by drift when rare, we arbitrarily took into account only haplotypes with a frequency higher than 0.01): i) loss of SI: when SC mutant haplotypes had invaded the population and all $S$-haplotypes were lost; ii) diversification: when the number of $S$-haplotypes reached $n+1=6$ and the frequency of SC haplotypes was lower than 0.1;

250 iii) undecided: When none of the two previously described events happened before $5.10^{5}$ generations. We estimated the probability of diversification by the number of runs where the event Diversification occurred among the one hundred runs performed for a given parameter set.

\section{Number of $S$-haplotypes at stationary state}

255 In order to study the effect of population structure on the number of haplotypes maintained at equilibrium in the metapopulation with the 2-steps mutation model, we determined the parameter range allowing $S$-haplotypes to diversify for four different pollen dispersal rates $d_{p}$ and three different mutation rates $\mu_{2}$. We ran simulations using parameter ranges where $d i$ 260 versification (as define above) occurred with probability $\geq 0.5$ (Fig. S5). Simulations were run as described in the previous section one hundred times for a given parameter set, with the exception that we let the metapopulation evolve for $10^{6}$ generations instead of stopping it when a diversification occurred. The parameters sets values were chosen such as the probability of diversification was high (see previous section). The number of functional $S$-haplotypes at the end of the simulations reflects the whole history of the population where SC and functional $S$-haplotypes can appear by random mutations. The average number of $S$-haplotypes at steady state $(\widehat{n})$ was estimated over all replicates for a given parameter set by calculating the number of $S$-haplotypes with a frequency $>0.01$ during the last 50,000 generations. In order to decouple the specific effect of population structure on the number of haplotypes generated by the diversification process from 
the effect of population structure on the probability of diversification we discarded cases where SI was lost or no diversification had occurred.

275 Interaction between the genetic architecture and population structure and its effect on diversification

In order to evaluate the effect of the genetic architecture underlying SI on the diversification in a structured population, we compared the average number of $S$-haplotypes at steady state under the 1-step vs. 2-steps mutational models. For this comparison to be meaningful, the number of $S$-haplotypes generated at equilibrium in a non-structured population with the same mutation rate must be the same in both models. In the 1-step mutation model, the rate at which new $S$-haplotypes appear is equal to the mutation rate $\mu_{1}$. In the 2 -steps mutation model, this rate is not straightforward since it depends on the frequency of SC mutants and on the mutation rate of the pistil and pollen genes $\mu_{2}$ (Gervais et al., 2011). We defined an effective mutation rate as the value $\mu_{1}$ such that the average number of $S$-haplotypes is similar in the 1-step vs. 2-steps mutation model $\left(\widehat{n}_{1} \approx \widehat{n}\right.$ i.e. the value of $\mu_{1}$ that gave the closest value of $\widehat{n}_{1}$ from $\widehat{n}$ ) in a non-subdivided population. We then ran simulations under both mutational models for the same parameter sets, including the effective mutation rate, for different levels of population structure. Comparing the number of $S$-haplotypes at steady state under both mutational models allowed us to isolate the specific effect of the interaction between population structure and genetic architecture.

\section{Scenarios of fitness valleys crossing in structured populations}

Population structure expands the number of scenarios by which new $S$ alleles can be formed, according to whether they arose in the same or different demes and whether the intermediate SC haplotype was maintained along with other SI haplotypes in the different demes. Fitness valleys can thus be crossed according to five different possible scenarios (summarized in Figure 2):

1. Local diversification - The two mutations occurred in the same deme and the ancestral haplotype was not lost from this deme. Under this scenario, the number of $S$-haplotypes increases at the deme scale, and consequently also at the metapopulation scale.

2. Local replacement - The two mutations occurred in the same deme but the ancestral haplotype was lost from this deme. Under this scenario, the number of $S$-haplotypes does not change at the deme scale, 
but it increases at the metapopulation scale (provided that the ancestral haplotype is present in at least one other deme).

3. Allodiversification - The two mutations occurred in different demes (i.e. at least one dispersal event of the intermediate SC haplotype occurred before the compensatory mutation) and the ancestral haplotype was conserved in the deme where the compensatory mutation occurred. Under this scenario, the number of $S$-haplotypes increases at the deme scale, and consequently also at the metapopulation scale.

4. Alloreplacement - The two mutations occurred in different demes (i.e. at least one dispersal event of the intermediate SC haplotype occurred before the compensatory mutation) but the ancestral haplotype was lost from the deme where the compensatory mutation occurred. Under this scenario, the number of $S$-haplotypes does not change at the deme scale, but it increases at the metapopulation scale.

5. Global replacement - The two mutations occurred in different demes but the ancestral haplotype was lost from the whole metapopulation. Under this scenario, the number of $S$-haplotypes does not change neither at the deme scale nor at the metapopulation scale.

The first four scenarios lead to a net global diversification (an increase in the number of $S$-haplotypes at the global scale; Fig. 2A, 2B, 2C and 2D) but not the Global replacement scenario where the new $S$-haplotype replaces its ancestor in the whole metapopulation (Fig. 2E).

To evaluate the relative frequency of these five scenarios in the dynamics of allelic diversification in a metapopulation we recorded the fate of every new SC haplotype during 1,000,000 generations in the 2-steps mutation model. We recorded the demes where the initial and the compensatory 335 mutation occurred and the frequency of the ancestral $S$-haplotype in each deme when the compensatory mutation occurred.

\section{Results}

In this study we asked three main questions: 1) does population structure affect the probability of diversification? 2) how does population structure affect the number of $S$-haplotypes relative to an isolated population, and if so, 3) what is the role of the 2-genes genetic architecture in determining these effects? 


\section{Population structure only slightly affects the probability of $S$-haplotype diversification}

We first ran a hundred replicates of simulation runs for varying parameter sets under a 2-steps mutational model, starting with an initial number of $S$-haplotypes $n=5$. We estimated the probability of diversification as the proportion of replicates with $n>5$ established $S$-haplotypes before the end of the simulation (Fig. 3).

Decreasing the pollen dispersal rate $d_{p}$ and hence increasing population subdivision shrunk the parameter regions where diversification was possible, and decreased the overall probability of diversification (Fig. 3). However, very strong levels of population structure are required to make this effect appreciable. Increasing the mutation rate $\mu$, (because more SC haplotypes are generated) expands the parameter region of diversification and decreases the effect of population structure in shrinking the parameter region of diversification (for $\mu=5 \times 10^{-6}$ the parameter region of diversification is halved between $d_{p}=0.8$ and $d_{p}=10^{-5}$ whereas for $\mu=5 \times 10^{-3}$ the effect is much weaker). For the lowest value of the mutation rate that we explored $\left(\mu=5 \times 10^{-7}\right)$, increasing pollen dispersal rate first expanded the parameter region allowing diversification and then shrunk it. This non-monotonous effect of population structure could be an artifact of the cut-off number of generations after which we determine the state of the population, as the mutation-selection-drift equilibrium takes longer to be reached for low mutation rates. In most cases, the differences between the less structured population $\left(d_{p}=0.8\right)$ and the most structured population $\left(d_{p}=10^{-8}\right)$ in the size of the parameter range allowing diversification are quantitatively similar to the differences between large and small non-structured populations $(N=5000$ vs. $N=1000)$. This led us to hypothesize that the shrinkage

370 of the diversification region with population structure is largely due to a reduction of the overall effective population size caused by a reduced pollen dispersal between demes.

\section{Parallel local replacements in different demes promote global diversification}

375 In order to study the effect of population structure on the number of $S$ haplotypes maintained in the metapopulation, we performed simulations for parameter values where the diversification frequency was higher than $50 \%$ (Fig. 3) for three values of the mutation rate $\mu$ and three values of the pollen dispersal rate $d_{p}$. (Fig. S5). We found that the average number of $S$ haplotypes at the metapopulation level at steady state $(\widehat{n})$ always increased 
with decreasing pollen migration rate $d_{p}$ (Fig. 4 and Fig. S6). The magnitude of the increase ranges from $5 \%$ to $92 \%$ depending on the combination of parameters. The effect was especially marked for very low dispersal rates $\left(d_{p}=10^{-8}\right)$ but was more subtle for higher dispersal rates $\left(d_{p}=10^{-2}\right.$ and $\left.d_{p}=10^{-5}\right)$. Our results thus suggest that population subdivision favors $S$-haplotype diversity, but only in highly structured metapopulations.

We then aimed at describing how the two successive mutations on a single haplotype occurred when new $S$-haplotypes were formed. We recorded whether the compensatory mutation appears in the same or in a different deme than the ancestral $S$-haplotype, and whether the ancestral $S$ haplotype was lost at the local (deme) or the global (metapopulation) scale (Fig. 5). The relative importance of the different fitness valley crossing scenarios differed sharply between largely unstructured $\left(d_{p}=0.8\right)$ and structured populations $\left(d_{p}=10^{-5}\right.$ and $\left.10^{-8}\right)$ (Fig. 5). In unstructured populations $\left(d_{p}=0.8\right)$, when the self-pollination rate was low $(\alpha \leq 0.6)$, emergence of new $S$-haplotypes was most of the time accompanied with global loss of the ancestral $S$-haplotype, with successive mutations occurring in different demes (global replacement). With higher self-pollination rates, global replacement became as frequent as allodiversification (i.e. the scenario in which the two mutations occur in different demes but the ancestral $S$-haplotype is conserved). This is due to the fact that SC haplotypes can more easily exclude their ancestral haplotype when they are still able to frequently outcross. Fitness valley crossings within demes (by local replacement or local diversification) represented a little less that $25 \%$ of the ob405 served fitness valleys crossing for all parameter values explored, i.e. in $75 \%$ of the cases, the first and second mutations occurred in different demes. In highly structured populations, in contrast, fitness valley crossings were almost exclusively due to within-demes events (local replacement and local diversification), and global replacements were rare. Our results thus

410 shows that population structure increases global $S$-haplotype diversity both because diversification and replacement take place in parallel in different demes, and because the loss of ancestral $S$-haplotypes at the scale of the metapopulation also becomes more rare.

\section{Interaction between population subdivision and genetic archi- 415 tecture increases $S$-haplotype diversity}

Our last goal was to check whether the genetic architecture underlying SI could change the effect of population structure on $S$-haplotype diversity. To achieve this, we compared the average $S$-haplotype diversity in structured 
populations under the 1-step vs. 2-steps mutational models. We determined the (effective) mutation rate of the 1-step mutational model under which the average number of $S$-haplotypes best approximated that under the 2 steps mutational model $\left(\widehat{n}_{1} \approx \widehat{n}\right)$ in an unstructured population. For all parameter sets we studied, we found that the 2-steps mutational model was more sensitive to population structure, consistently producing new $S$ haplotypes at a higher rate than the 1 -step mutational model $\left(\widehat{n} / \widehat{n}_{1} \geq 1\right.$ in Fig. 6). The contrast between the two mutational models was higher when the dispersal rate decreased, resulting in a higher number of $S$-haplotypes under the 2-steps model than under the 1-step model. The number of $S$ haplotypes can be twice higher under the 2-steps than 1-step mutational model even though our simulations were calibrated so that the two mutation models behaved identically in unstructured populations. Our results thus showed that in structured populations, a more complex genetic architecture underlying SI can favor the emergence of $S$-haplotype diversity even the effective mutation rates are identical.

\section{Discussion}

\section{Population structure enhances $S$-haplotypes diversification}

Our results show that population structure monotonically increases the $S$ haplotype diversity at the metapopulation level, although it also decreases the probability of $S$-haplotype diversification overall. Schierup (1998) showed that, in a 1-step mutation model, the $S$-allele diversity at the metapopulation level varies non-monotonically with subdivision, because: 1) $S$-alleles can be lost by chance in isolated demes (genetic drift), 2) balancing selection increases the invasion probability of new $S$-alleles when the local $S$-allele diversity is low, and 3) dispersal homogenizes $S$-allele diversity among demes. In the 2-steps mutation model, our results show that limited dispersal enhances global diversity. While the same phenomena at the local scale also apply, this genetic architecture modifies the interactions between underlying mechanisms. Indeed, even after controlling for different effective mutations rates under the 1-step vs. 2-steps mutation models, $S$-haplotype diversity was always higher under the 2-steps than the 1-step mutation model (Fig. 6). This suggests that crossing fitness valleys through partial self-fertilization modifies the interactions between selection and dispersal, ultimately affecting the diversity of $S$-haplotypes.

Two mechanisms can explain why population structure increases the $S$-haplotype diversity. First, as shown by Gervais et al. (2011), the diver- 
sification rate is higher when the number of haplotypes is low. Since the relative fitness of SC haplotypes (compared to $S$-haplotypes) is higher when the number of $S$-haplotypes is low, their expected frequency is also higher, which makes the appearance of a compensatory mutation more probable. Population structure thus enhances $S$-haplotype diversity because locally the number of $S$-haplotypes is decreased, causing diversification to be faster at the local scale. This is supported by our observations that the occurrence of local diversification events increases with decreasing dispersal. Second, the relative importance of scenarios by which fitness valleys were crossed varies with population structure. In a non-structured population, new $S$ haplotypes emerge either by a replacement or a diversification (Gervais et al., 2011). In a structured population, global diversification also comes from events of local replacement. As replacements taking place in different demes likely affects different $S$-haplotypes, different $S$-haplotypes will be lost and replaced locally. Replacement occurring in different demes thus increases $S$-haplotype diversity at the global scale.

Overall, can limited dispersal help fill the gap between observed vs. expected $S$-haplotype diversity? While we do see an increase in the number of $S$-haplotypes caused by population structure, even for very small dispersal rates, the increase is quantitatively moderate, and still far from explaining the very large $S$-haplotype diversity observed in natural populations (from a few dozens to $>100$ haplotypes, Lawrence 2000). Since population isolation tends to enhance the local diversification dynamics, the large diversity in natural populations might be the result of the complex demographic his480 tory of SI species, where populations have several times fragmented and merged, expanded and shrunk, lost and gained pollinators, or exchanged $S$-haplotypes with closely related species. It may also be that other mechanisms contribute to the rise of new $S$-haplotypes in nature. For example, Sakai (2016) proposed that $S$-haplotypes can have been generated from SC 485 populations by progressive optimization of the self-recognition capacity between $S$-haplotypes that initially have incomplete recognition and rejection. He found that this model can generate up to 40-50 haplotypes in an unstructured SC population within a few hundred generations. How population structure would affect $S$-haplotype diversity in this model is an open question. 


\section{Fitness valley crossing in structured populations under bal- ancing selection}

The crossing of fitness valleys has been studied by population genetics models under directional selection, where the fitness landscape is static as it results from fixed parameters. Balancing selection makes the fitness landscape variable because its shape depends on the frequency of the different haplotypes. Fitness valley Crossings under directional selection can occur through two mechanisms (Weissman et al., 2009): by stochastic tunnelling, where intermediate deleterious genotypes segregate in low frequency because of recurrent mutations; or by sequential fixation, where the intermediate genotype first gets fixed and then the advantageous genotype arises by mutation and goes to fixation. Population structure can facilitate the crossing of fitness valleys under directional selection, a phenomenon interpreted as resulting from "competitive assortment" (Bitbol and Schwab, 2014; Komarova et al., 2014; Cooper and Kerr, 2016; McLaren, 2016). As dispersal is limited and competition between genotypes occurs locally, local kinship decreases the relative disadvantage of intermediate deleterious genotypes relatively to their wild-type counterparts. The frequency of intermediate genotypes is thus higher, they can even possibly get fixed, which facilitates stochastic tunnelling and sequential fixation.

In our analysis, the temporary segregation of SC intermediate haplotypes in local demes due to limited dispersal can be interpreted as competitive assortment and may thus facilitate $S$-haplotype diversification through stochastic tunnelling. Diversification is expected to be proportional to the residence time of SC intermediate haplotypes within demes, because the longer the residence time, the higher the probability that a compensatory mutation will occur. The residence time of SC intermediate haplotypes depends on selection acting on SC intermediate haplotypes (depending on the relative importance of inbreeding depression and mate availability). Competitive assortment mitigates the effect of selection against SC intermediate haplotypes because the mate availability advantage increases when the local kinship is higher (a higher kinship means a lower number of $S$-haplotypes). In contrast, local sequential fixation (sequential fixation in a single deme) is unlikely to be common. Indeed, the coexistence of a deme where a SC variant is fixed along with other demes where SI is conserved is never observed in a finite islands population (see also Brom et al., 2020). This is because, neglecting stochastic fixation, if the conditions are met for a SC haplotype to fix in one deme, SC haplotypes will also tend to get fixed in all other demes of the metapopulation. Overall, fitness valley crossing in the case 
of $S$-haplotypes diversification in a subdivided population is mostly due to stochastic tunnelling rather than sequential fixation.

A series of models (Bod'ová et al., 2018; Harkness et al., 2019; Harkness and Brandvain, 2021) have focused on a deeply rooted SI system in Angiosperms based on non-self recognition of the pollen ( Fujii et al., 2016). The mutational pathway underlying the emergence of new $S$-haplotypes in such systems is different than the one we supposed here. Bod'ová et al. (2018) found that in such a system new $S$-haplotypes can emerge through a variety of pathways, in particular frequently by a SC intermediate haplotype (i.e. a fitness valley crossing). The SC intermediate pathway becomes more likely compared to other pathways when the number of specificities increases and when the mutation rate decreases. How population structure might affect diversity in non-self recognition SI systems is an open question.

Overall, our model suggests that the residence time of SC intermediate haplotypes is essential for $S$-haplotype diversification. We can speculate that new $S$-haplotypes are most likely to occur in small isolated demes, or in populations at the margins of species range where SI is most frequently lost e.g. Arabidopsis lyrata (Griffin and Willi, 2014). Observations in such populations are now needed to test the models' predictions.

\section{How would a SI species evolve after fragmentation or pollina- tors loss?}

It is not yet totally understood why some species have lost SI while others have not, even among sister species. It is possible that different demographic histories, including histories of habitat fragmentation and pollinators loss may lead to these different outcomes. Indeed, Brom et al. (2020) predicted that decreasing dispersal rate in a subdivided population should facilitate the loss of SI because the local effective number of $S$-haplotypes is decreased. Encinas-Viso et al. (2020) further showed that the loss of SI can also occur during range expansion in the range margins and extend to the core population under certain conditions. However, here, we showed that popuation subdivision makes $S$-haplotype diversification both faster and easier. Hence, we argue that the evolutionary dynamics of a SI species during range expansion, or which recently suffered a fragmentation or pollinators loss is bistable. Either SI will be lost in the short run, or SI will diversify again rapidly. In a nutshell, the loss of SI and SI diversification can be viewed as conflicting processes, and the evolutionary trajectory followed by a species is the result of the race between both processes. A better understanding of how demographic processes and genetic mechanisms affect the balance 
between SI diversification and SI loss could help predict whether SI loss or SI rescue is the most likely scenario after range expansion, fragmentation, population bottleneck, or the loss of a pollinator.

\section{References}

Barrett, S. C. (2002). The evolution of plant sexual diversity. Nature Reviews Genetics, 3(4):274-284.

Billiard, S., Lopez-Villavicencio, M., Devier, B., Hood, M. E., Fairhead, C., and Giraud, T. (2011). Having sex, yes, but with whom? Inferences from fungi on the evolution of anisogamy and mating types. Biological Reviews, 86(2):421-442.

Bitbol, A.-F. and Schwab, D. J. (2014). Quantifying the role of population subdivision in evolution on rugged fitness landscapes. PLoS computational biology, 10(8):e1003778.

Bod'ová, K., Priklopil, T., Field, D. L., Barton, N. H., and Pickup, M. (2018). Evolutionary pathways for the generation of new selfincompatibility haplotypes in a non-self recognition system. Genetics, 209(3):861-883.

Bovier, A., Coquille, L., and Smadi, C. (2019). Crossing a fitness valley as a metastable transition in a stochastic population model. Ann. Appl. Probab., 29(6):3541-3589.

Bowen, D. G. and Walker, C. M. (2005). Mutational escape from CD8+T cell immunity: HCV evolution, from chimpanzees to man. The Journal of Experimental Medicine, 201(11):1709-1714.

Brom, T., Castric, V., and Billiard, S. (2020). Breakdown of gametophytic self-incompatibility in subdivided populations. Evolution, 74:270-282.

Burch, C. L. and Chao, L. (2000). Evolvability of an RNA virus is determined by its mutational neighbourhood. Nature, 406(6796):625-628.

Castric, V. and Vekemans, X. (2004). Plant self-incompatibility in natural populations: a critical assessment of recent theoretical and empirical advances. Molecular Ecology, 13(10):2873-2889.

Cooper, J. D. and Kerr, B. (2016). Evolution at 'sutures' and 'centers': recombination can aid adaptation of spatially structured populations on rugged fitness landscapes. PLoS computational biology, 12(12):e1005247. 
de Visser, J. A. G. M. and Krug, J. (2014). Empirical fitness landscapes and the predictability of evolution. Nature Reviews Genetics, 15(7):480-490.

Encinas-Viso, F., Young, A., and Pannell, J. (2020). The loss of selfincompatibility in a range expansion. Journal of Evolutionary Biology, 33(9):1235-1244.

Fernandez, C. S., Stratov, I., Rose, R. D., Walsh, K., Dale, C. J., Smith, M. Z., Agy, M. B., Hu, S.-l., Krebs, K., Watkins, D. I., Connor, D. H. O., Davenport, M. P., Kent, S. J., and Irol, J. V. (2005). Rapid viral escape at an immunodominant simian-human Immunodeficiency virus cytotoxic T-lymphocyte epitope exacts a dramatic fitness cost. Journal of Virology, 79(9):5721-5731.

Fujii, S., Kubo, K. I., and Takayama, S. (2016). Non-self- and self-recognition models in plant self-incompatibility. Nature Plants, $2(9): 16130$.

615 Gervais, C., Awad, D. A., Roze, D., Castric, V., and Billiard, S. (2014). Genetic architecture of inbreeding depression and the maintenance of gametophytic self-incompatibility. Evolution, 68(11):3317-3324.

Gervais, C., Castric, V., Ressayre, A., and Billiard, S. (2011). Origin and diversification dynamics of self-incompatibility haplotypes. Genetics, 188(3):625-636.

Griffin, P. C. and Willi, Y. (2014). Evolutionary shifts to self-fertilisation restricted to geographic range margins in North American Arabidopsis lyrata. Ecology Letters, 17(4):484-490.

Harkness, A. and Brandvain, Y. (2021). Non-self recognition-based selfincompatibility can alternatively promote or prevent introgression. New Phytologist.

Harkness, A., Goldberg, E. E., and Brandvain, Y. (2019). Diversification or collapse of self-incompatibility haplotypes as outcomes of evolutionary rescue. bioRxiv, pages 1-37.

630 Igic, B., Lande, R., and Kohn, J. R. (2008). Loss of self-incompatibility and its evolutionary consequences. Journal of Plant Sciences, 169(1):93-104.

Komarova, N. L., Shahriyari, L., and Wodarz, D. (2014). Complex role of space in the crossing of fitness valleys by asexual populations. J. R. Soc. Interface, 11(95):20140014. 
635 Korona, R., Nakatsu, C. H., Forney, L. J., and Lenskit, R. E. (1994). Evidence for multiple adaptive peaks from populations of bacteria evolving in a structured habitat. Proceedings of the National Academy of Sciences, 91(19):9037-9041.

Kvitek, D. J. and Sherlock, G. (2011). Reciprocal sign epistasis between fre640 quently experimentally evolved adaptive mutations causes a rugged fitness landscape. PLoS Genetics, 7(4):e1002056.

Lawrence, M. J. (2000). Population genetics of the homomorphic selfincompatibility polymorphisms in flowering plants. Annals of Botany, $85: 221-226$.

645 McCubbin, A. G. and Kao, T.-H. (2000). Molecular recognition and response in pollen and pistil interactions. Annu. Rev. Cell Dev. Biol, 16(1):333-364.

McLaren, M. (2016). Fitness-valley crossing in subdivided asexual populations. bioRxiv.

Sakai, S. (2016). How have self-incompatibility haplotypes diversified? Generation of new haplotypes during the evolution of self-incompatibility from self-compatibility. The American Naturalist, 188(2):163-174.

Salverda, M. L. M., Dellus, E., Gorter, F. A., Debets, A. J. M., Oost, J. V. D., Rolf, F., Tawfik, D. S., and Visser, J. A. G. M. D. (2011). Initial mutations direct alternative pathways of protein evolution. PLoS Genetics, 7(3):e1001321.

Schierup, M. H. (1998). The number of self-incompatibility alleles in a finite, subdivided population. Genetics, 149(2):1153-1162.

Schierup, M. H., Vekemans, X., and Charlesworth, D. (2000). The effect of subdivision on variation at multi-allelic loci under balancing selection. Genetics Researchics, 76(1):51-62.

Shih, A. C.-c., Hsiao, T.-c., Ho, M.-s., and Li, W.-h. (2007). Simultaneous amino acid substitutions at antigenic sites drive influenza A hemagglutinin evolution. Proceedings of the National Academy of Sciences, 104(15):62836288 .

Uyenoyama, M. K., Zhang, Y., and Newbigin, E. (2001). On the origin of self-incompatibility haplotypes: transition through self-compatible intermediates. Genetics, 157(4):1805-1817. 
Vos, M. G. J. D., Dawid, A., Sunderlikova, V., and Tans, S. J. (2015). Breaking evolutionary constraint with a tradeoff ratchet. Proceedings of the National Academy of Sciences, 112(48):4906-4911.

Weinreich, D. M., Delaney, N. F., DePristo, M. A., and Hartl, D. L. (2006). Darwinian evolution can follow only very few mutational paths to fitter proteins. Science, 312(5770):111-114.

Weissman, D. B., Desai, M. M., Fisher, D. S., and Feldman, M. W. (2009). The rate at which asexual populations cross fitness valleys. Theoretical Population Biology, 75(4):286-300.

Weissman, D. B., Feldman, M. W., and Fisher, D. S. (2010). The rate of fitness-valley crossing in sexual populations. Genetics, 186:1389-1410.

Whitlock, M. C., Phillips, P. C., Moore, F. B., and Tonsor, S. J. (1995). 680 Multiple fitness peaks and epistasis. Annual Review of Ecology and Systematics, 26(1):601-629.

Wright, S. (1932). The role of mutation, inbreeding, crossbreeding, and selection in evolution. Proceedings of the Sixth International Congress on Genetics, 1:356-366.

685 Wright, S. (1939). The distribution of self-sterility alleles in populations. Genetics, 24:538-552.

\section{Acknowledgements}

This work was funded by the European Research Council (NOVEL project, grant \#648321). The authors also thank the Région Hauts-de-France, the 690 Ministère de l'Enseignement Supérieur et de la Recherche (CPER Climibio), and the European Fund for Regional Economic Development for their financial support. 


\section{Box 1}

The question of $S$-haplotype emergence is the following: how is it possible to go from $n$ to $n+1 S$-haplotypes segregating in a population, given an intermediate SC haplotype which can have a fitness lower or higher than its ancestral $\left(S_{n}\right)$ and derived new $\left(S_{n+1}\right) S$-haplotypes depending on the cost of inbreeding depression relative to the strength of negative frequency dependent selection (Fig. S1A, B and C).

The number of $S$-haplotypes increases when a fitness valley is crossed either because the SC intermediate haplotype has a fitness lower than both $S_{n}$ and $S_{n+1}$ (Fig. S1B), or because $S_{n+1}$ has a higher fitness than $S_{n}$ when it co-occurs with the SC intermediate haplotypes and thus $S_{n}$ is lost (Fig. S1C) Uyenoyama et al. (2001) and Gervais et al. (2011)) showed that fitness valley crossing can be achieved by two mechanisms: either because the ancestral $S$-haplotype $S_{n}$ and the intermediate SC haplotype can stably coexist under negative frequency dependent selection, or because haplotypes with the lowest fitness can be maintained in the population long enough because of genetic drift. Genetic drift is thus an important mechanism allowing fitness valley crossing under either directional (Weissman et al., 2009) or negative frequency dependent (Gervais et al., 2011) selection. 
A
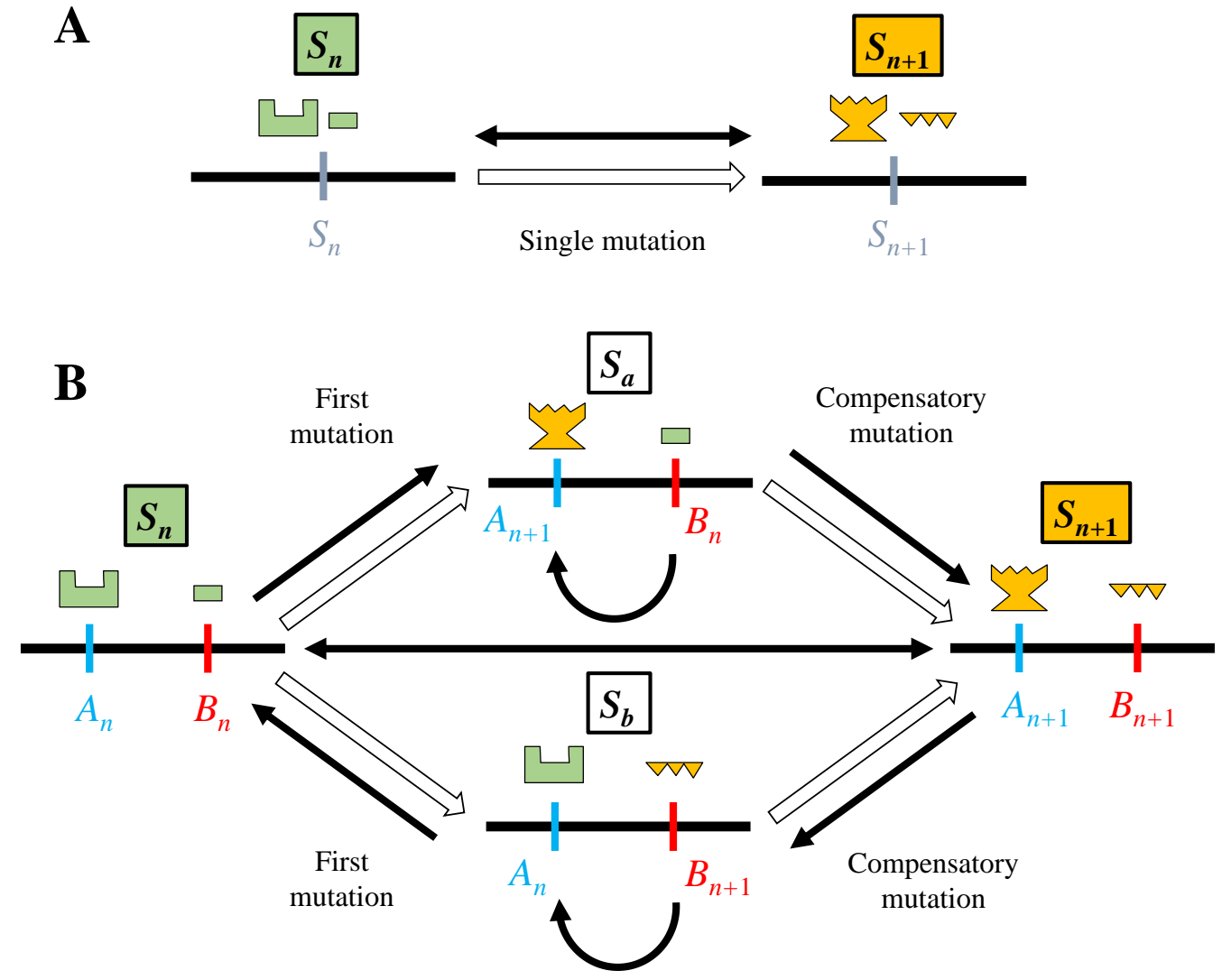

Fig. 1: Mutational pathway by which new haplotypes are created in the 1-step mutation model (A) and in the 2-steps mutation model (B; Uyenoyama et al., 2001; Gervais et al., 2011). Horizontal black lines represent chromosomes carrying the $S$-haplotype which is composed of just one gene in the 1-step mutation model and of two genes in the 2-steps mutation model with gene $A$ as the female determinant (blue vertical lines) and gene $B$ as the male determinant (red vertical lines). $S_{n}$ is the ancestral $S$ haplotype, $S_{n+1}$ the new $S$ haplotype, $S_{a}$ the pistil-part mutant SC haplotype and $S_{b}$ the pollen-part mutant $\mathrm{SC}$ haplotype. Incompatible specificities are represented by matching shapes and identical colours. White arrows represent mutation steps and black arrows indicate that the pollen carrying the haplotype at the base of the arrow can pollinate the pistil carrying the haplotype at the tip of the arrow. Double black arrows indicate mutual compatibility between haplotypes. Modified from Uyenoyama et al. (2001). 


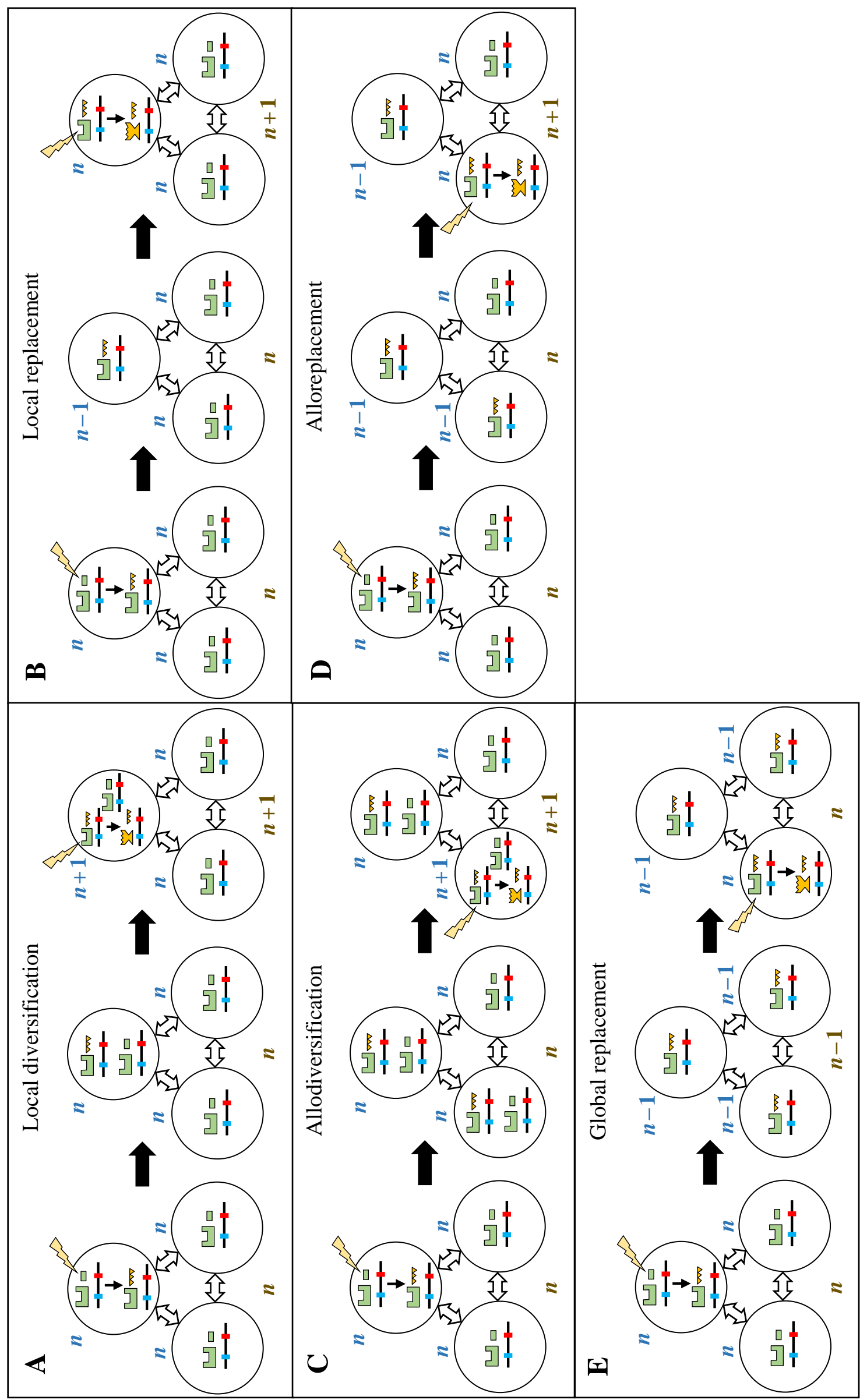

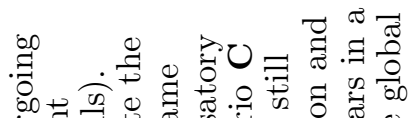

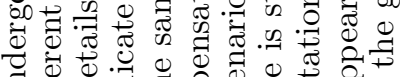

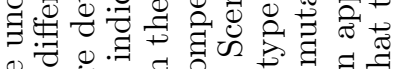

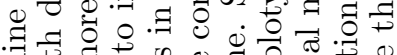

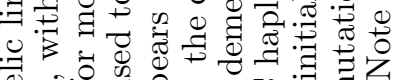
政语 .

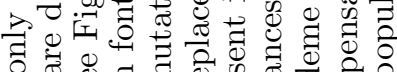

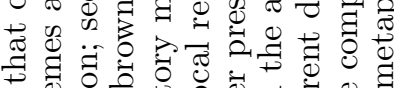

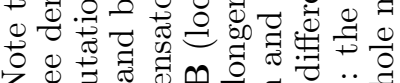
乙)

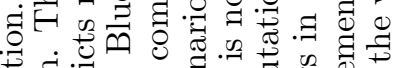
密. $\frac{0}{3}+口$ 论

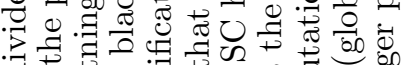
. क .

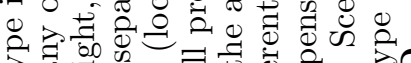

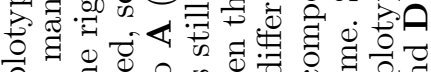

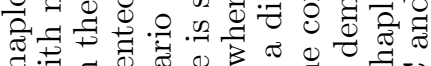

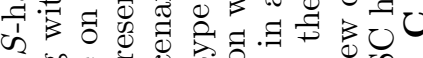

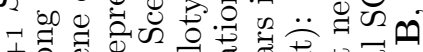

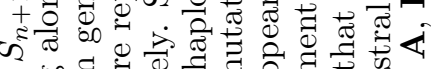

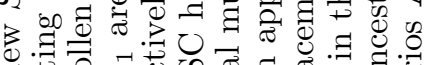

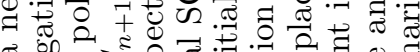

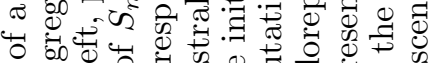

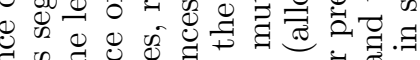
月. 然.

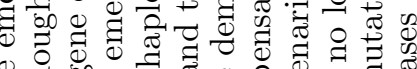
东

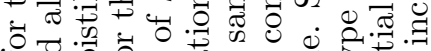
o

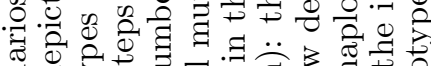

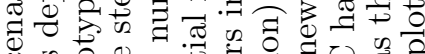

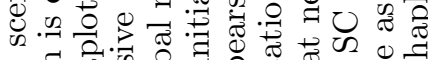

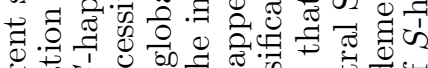

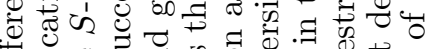

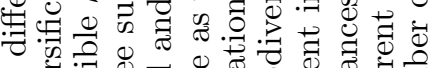

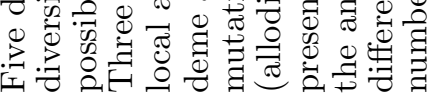
$\ddot{\sim}$ 


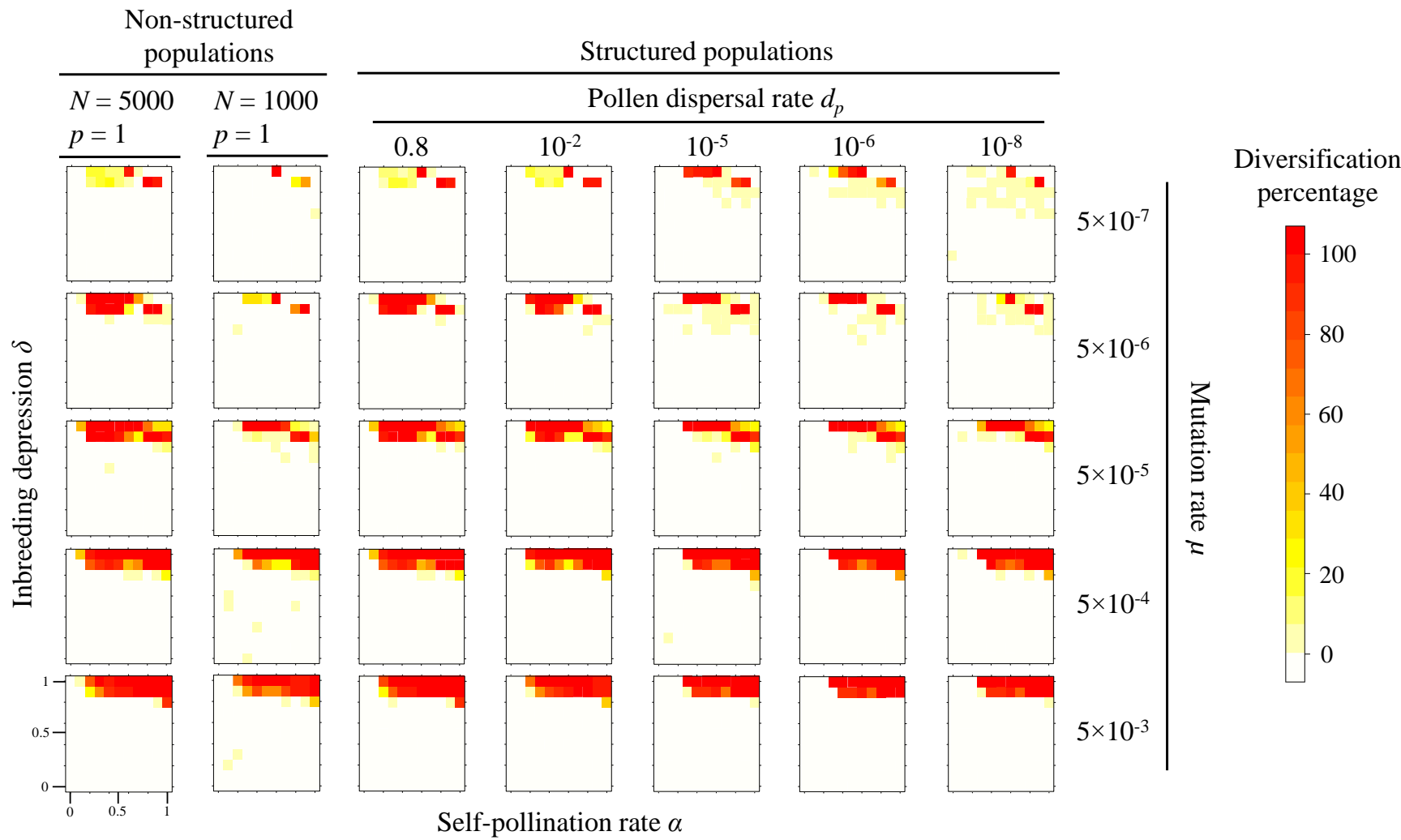

Fig. 3: Diversification of $S$-haplotypes as a function of the mutation rate $\mu$ and the pollen dispersal rate $d_{p}$. The metapopulation is composed of $p=5$ demes containing 1,000 individuals each, except the two leftmost columns where the population is non-structured: a single deme of size 1,000 or 5,000. Color scaling on the right refers to the percentage of simulation runs among 100 replicates, for a given parameters set, where the total number of $S$-haplotypes increased in the metapopulation (starting with an initial number $n=5$ ). 


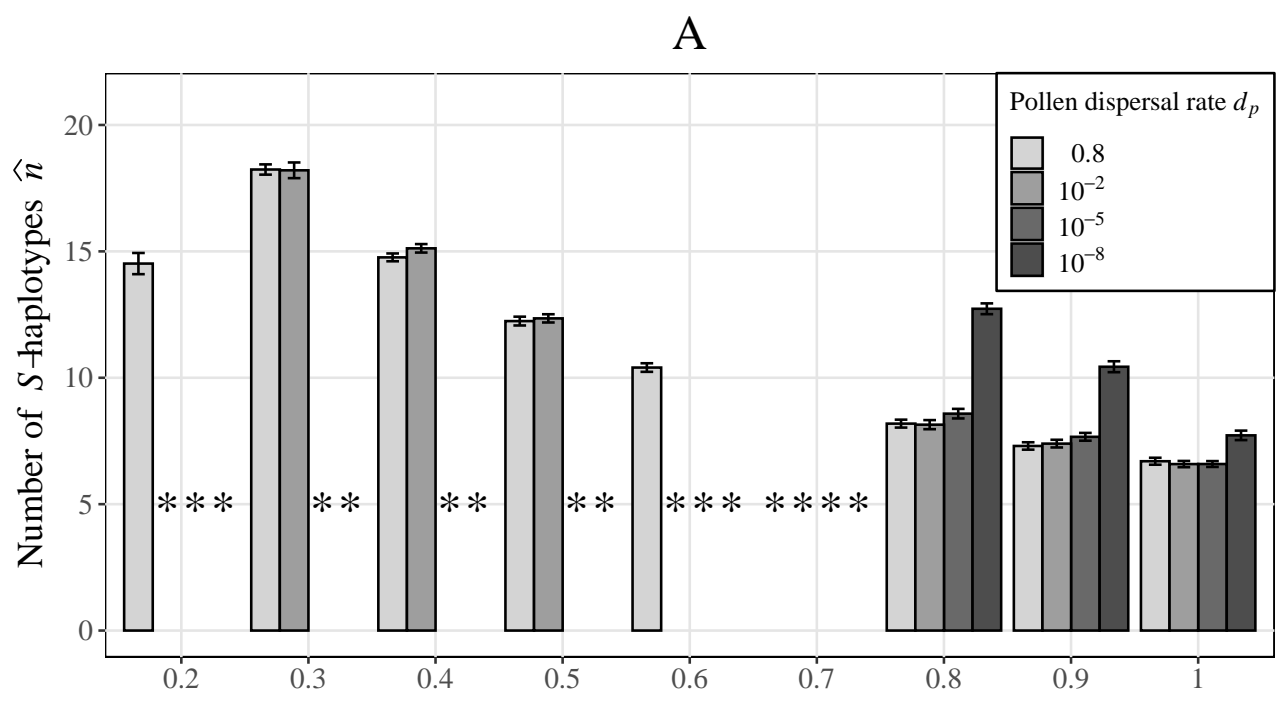

B

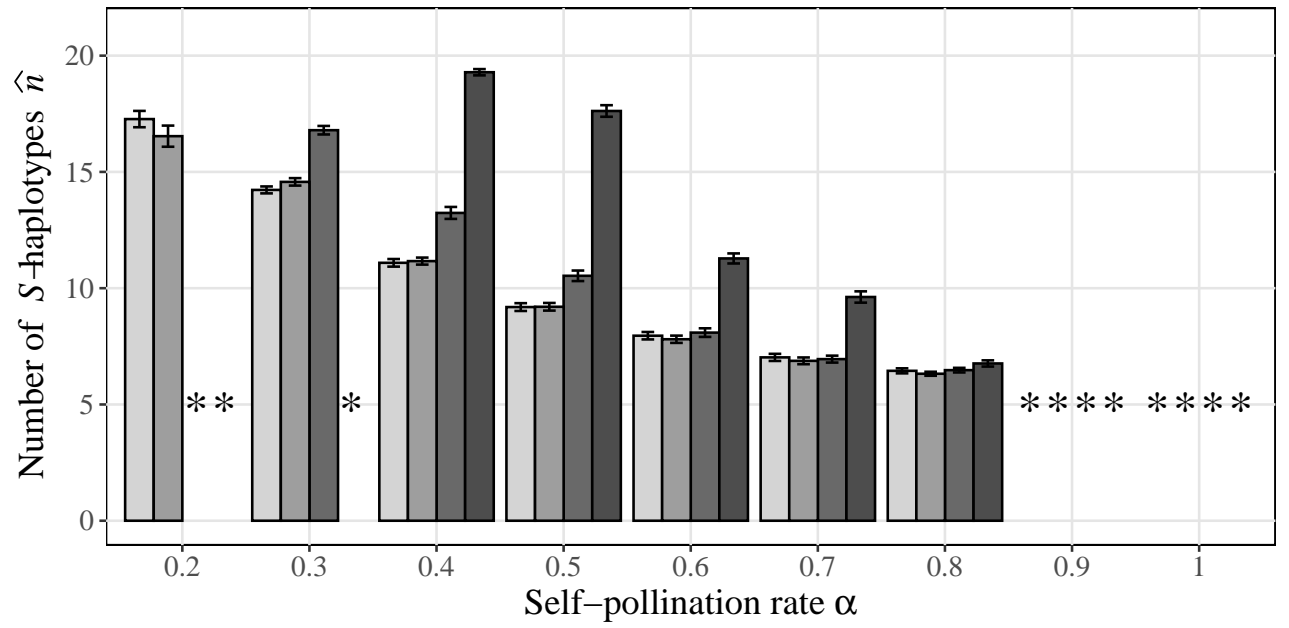

Fig. 4: Average number and $95 \%$ CI of $S$-haplotypes at steady state as a function of the self-pollination rate $\alpha$ and for different pollen dispersal rates $d_{p}$. An asterisk indicates a combination of parameters where diversification is too rare to be considered (inferior to $50 \%$ of diversification i.e. regions surrounded in black in Fig. S5). Parameters: $\mu_{2}=5 \times 10^{-5}$, $N=5000, p=5, n=5$ and $k=20$. A: $\delta=1 ; \mathrm{B}: \delta=0.9$. 
bioRxiv preprint doi: https://doi.org/10.1101/2021.11.20.469375; this version posted November 20, 2021. The copyright holder for this

preprint (which was not certified by peer review) is the author/funder, who has granted bioRxiv a license to display the preprint in perpetuity. It is made available under aCC-BY 4.0 International license.

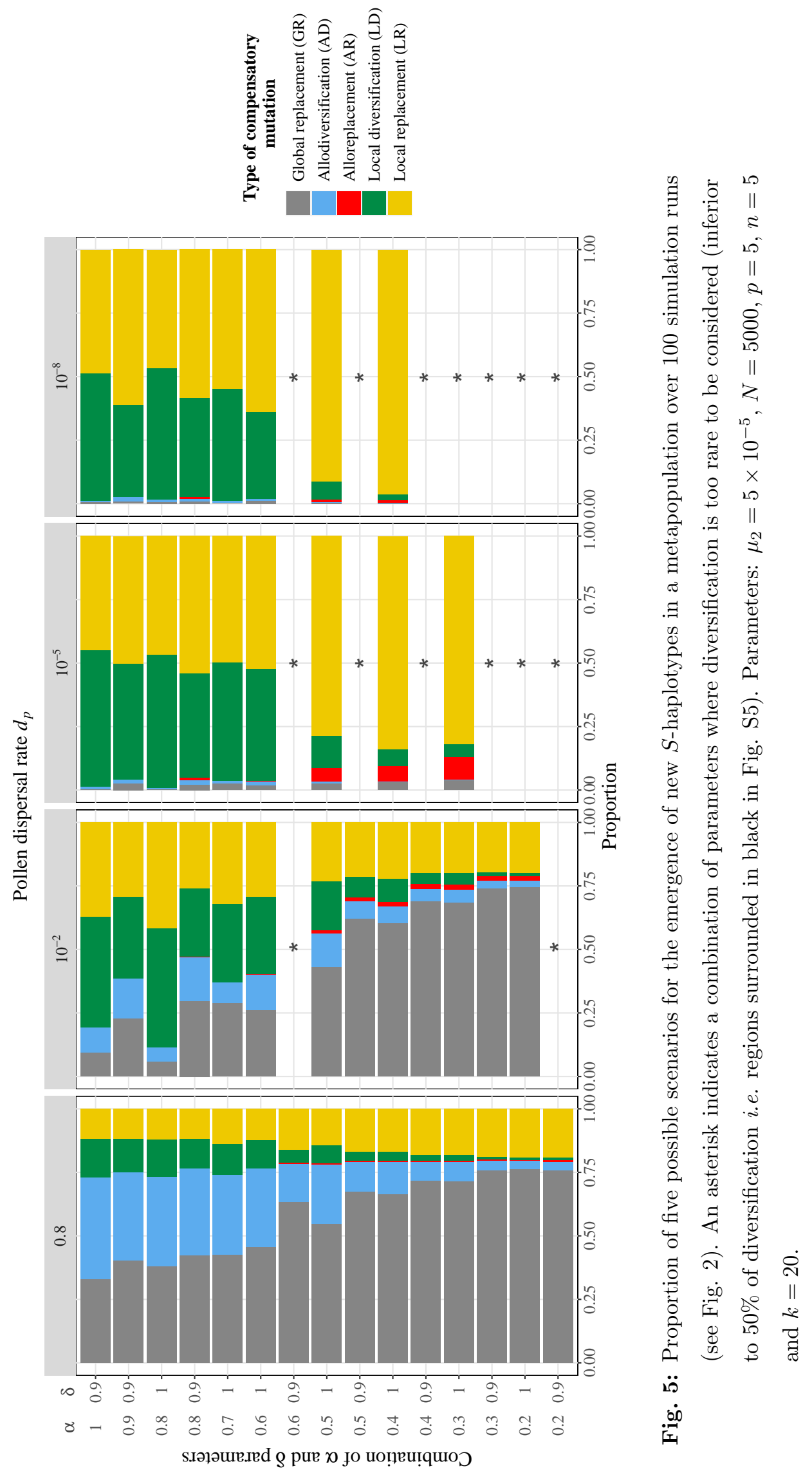




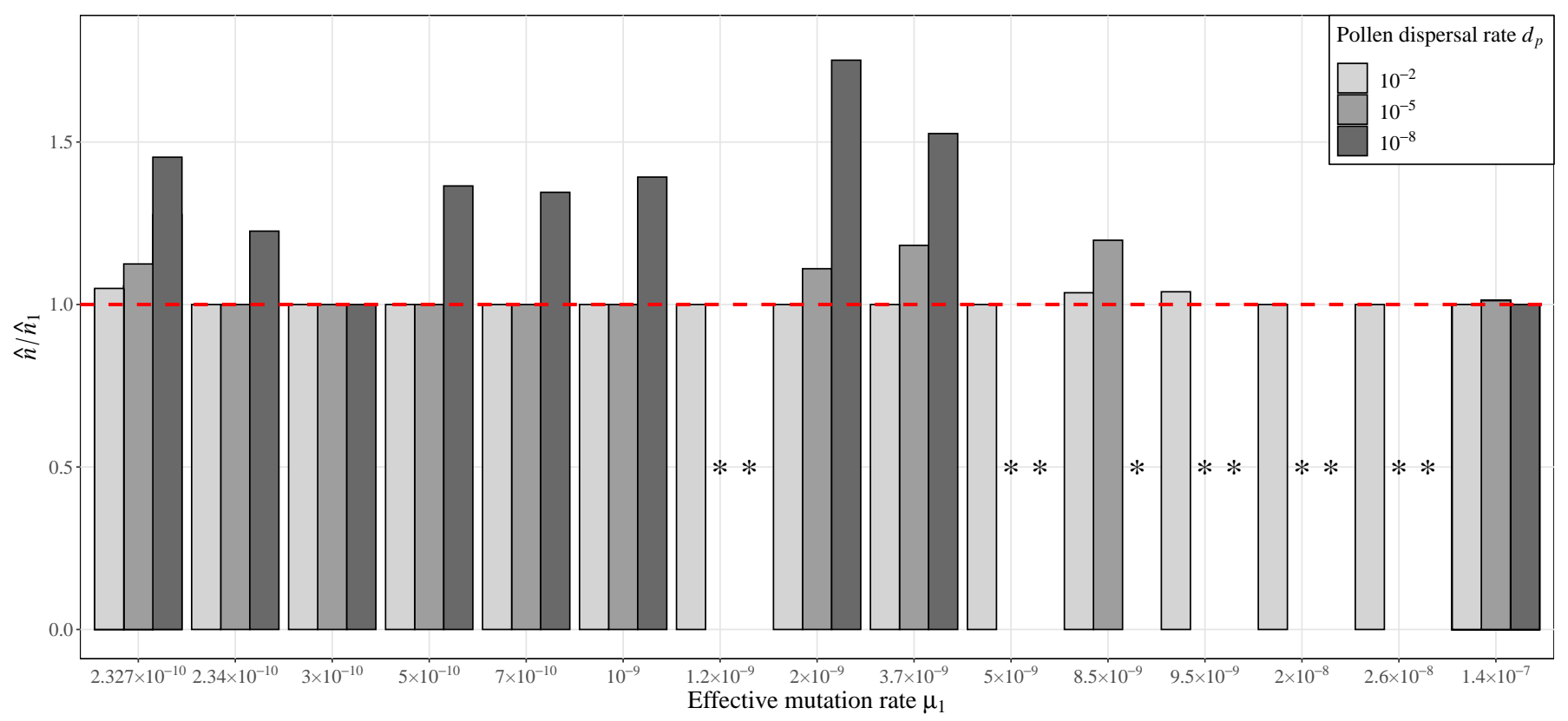

Fig. 6: Ratio of the number of $S$-haplotypes at steady state between the 2-steps vs. 1-step mutation models $\widehat{n} / \widehat{n}_{1}$ for a given effective mutation rate $\mu_{1}$ (see text for details). The red dashed line represents a ratio of 1 (with a $95 \% \mathrm{CI}$ ): in other words bars higher than the red dashed line mean that the number of $S$-haplotypes at equilibrium is significantly higher under the 2-steps than the 1-step mutation model even though the effective mutation rate is similar. An asterisk indicates a combination of parameters where diversification is too rare to be considered (inferior to $50 \%$ of diversification i.e. regions surrounded in black in Fig. S5). 

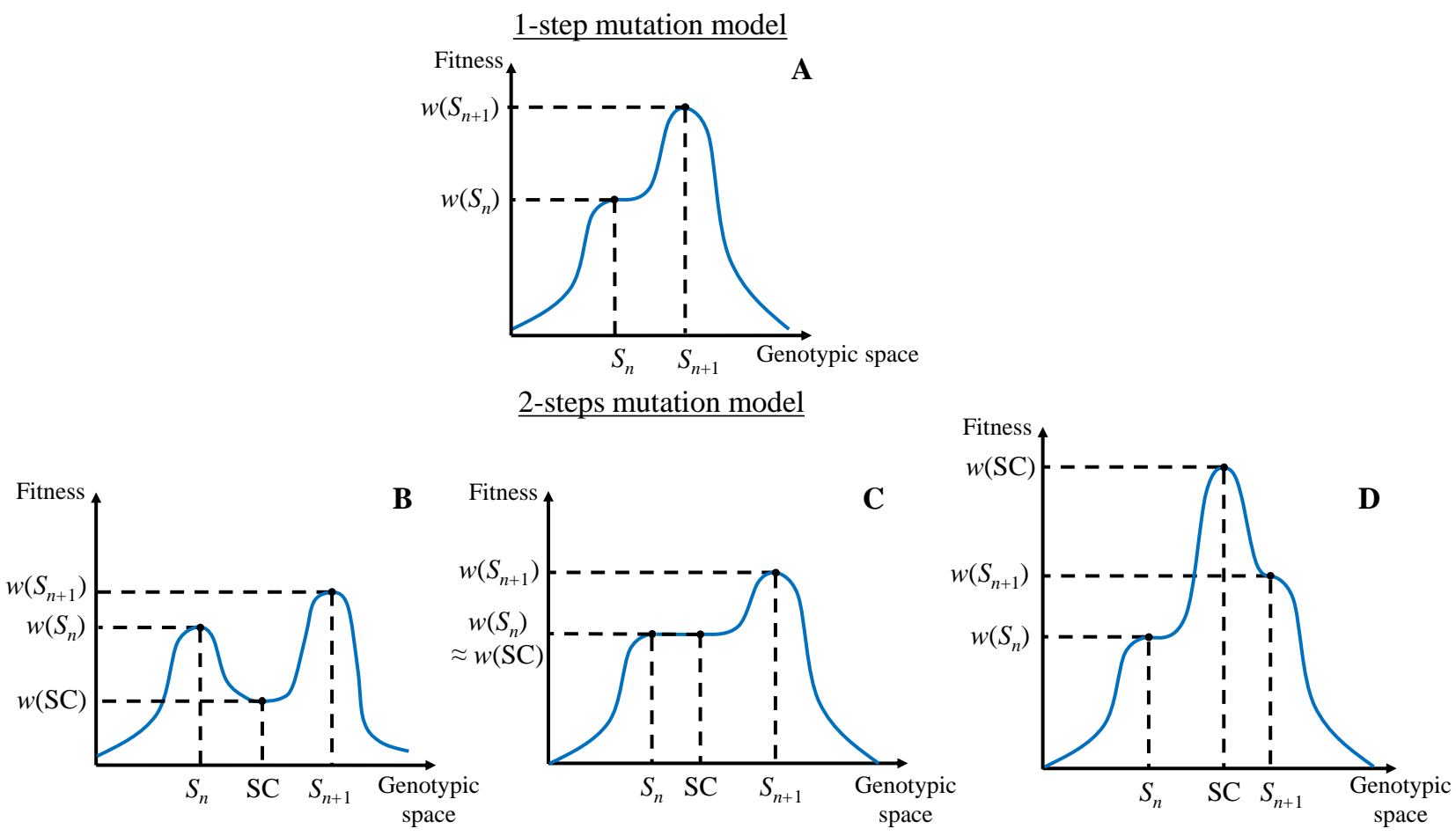

Fig. S1: Fitness landscapes when a new $S$-haplotype $S_{n+1}$ emerges under $(\mathbf{A})$ a 1-step or $(\mathbf{B}, \mathbf{C}, \mathbf{D})$ a 2-steps mutation model. X-axis: simplified 1D genotypic space where: $S_{n}$ represents the $n$ $S$-haplotypes in the population before the emergence of the new $S$-haplotype $S_{n+1}$; and $S_{b}$ represents a self-compatible intermediate haplotype in a 2-steps mutation model (see text for further details). Y-axis: relative fitness of each haplotype assuming that a new $S$-haplotype $S_{n+1}$ emerges at low frequency into a resident population, at mutation-drift-selection equilibrium, composed of $n S$-haplotypes with identical frequencies and a self-compatible intermediate haplotype. Because of negative frequency dependent selection, $S_{n+1}$ has a higher fitness on the landscape than the other $S_{n}$ haplotypes. The self-compatible intermediate haplotype can have three different positions on the fitness landscape: (B) it has either a fitness lower than all $S$-haplotypes; or it is present in the population because of recurrent mutation and/or genetic drift; or the emergence of a new $S$-haplotype $S_{n+1}$ needs the crossing of a fitness valley (stochastic tunneling); (C) The self-compatible intermediate haplotype coexists with $S_{n}$ haplotypes at equilibrium (they have the same fitness); the emergence of the new $S$-haplotype $S_{n+1}$ needs the crossing of a flat landscape; (D) The relative fitness of the self-compatible intermediate haplotype is higher than all $S$-haplotypes (including the new emerging $S_{n+1}$ ); the self-compatible intermediate haplotype will eventually go to fixation and the self-incompatibility system will be lost. 


\section{Diversification percentage}

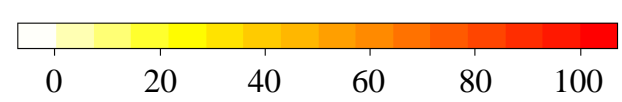
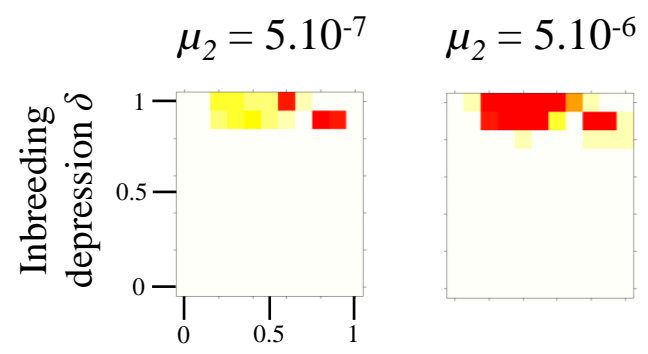

$$
\mu_{2}=5.10^{-5}
$$

$$
\mu_{2}=5 \cdot 10^{-4}
$$$$
\mu_{2}=5.10^{-3}
$$
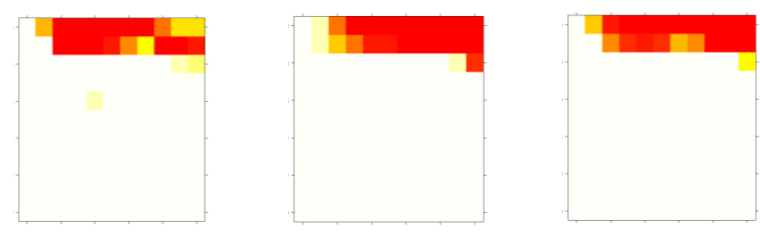

\section{Self-pollination rate $\alpha$}

Fig. S2: Parameter regions allowing diversification of $S$-haplotypes - run with our simulation program of the 2-steps mutation model - as a function of the mutation rate $\mu_{2}$ for a non-structured population. The colour range indicates the diversification percentage (the number of times diversification occurred over 100 replicates) and goes from white, meaning no diversification occurred, to dark red, meaning diversification always occurred. Each grid cell corresponds to 100 replicates. For all simulations $n=5, k=20, N=5000, p=5$. 


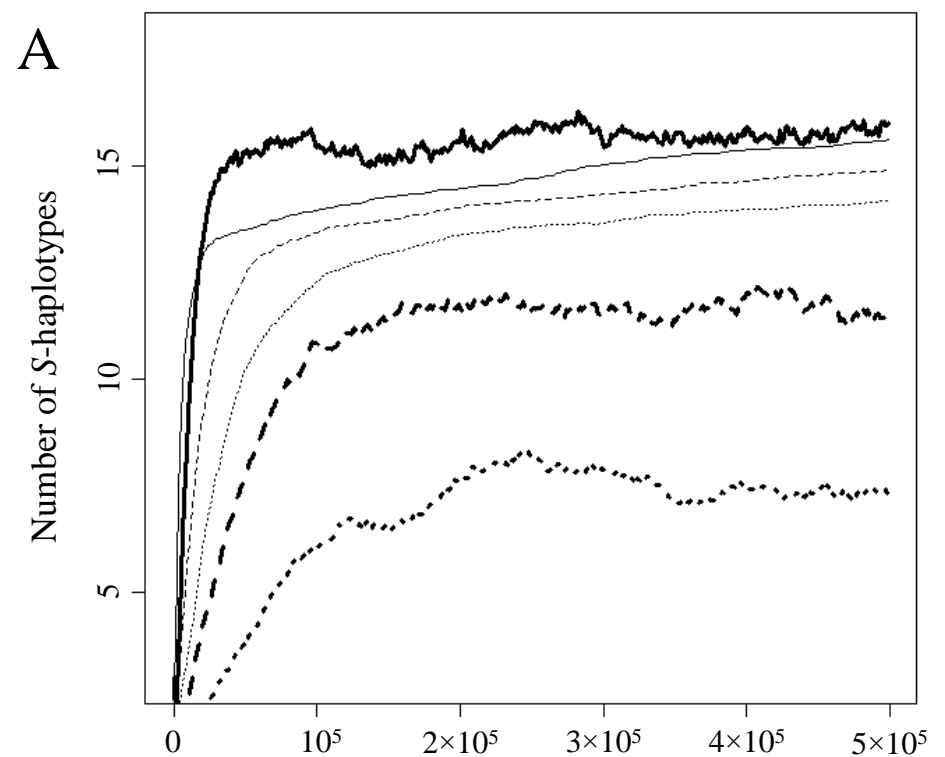

$$
\begin{aligned}
-\alpha & \alpha=0.2, k=20 \\
-\quad & \alpha=0.2, k=100 \\
\ldots \ldots & \alpha=0.2, k=200 \\
-\quad & \alpha=0.4, k=20 \\
--- & \alpha=0.4, k=100 \\
\cdots \cdots & \alpha=0.4, k=200
\end{aligned}
$$
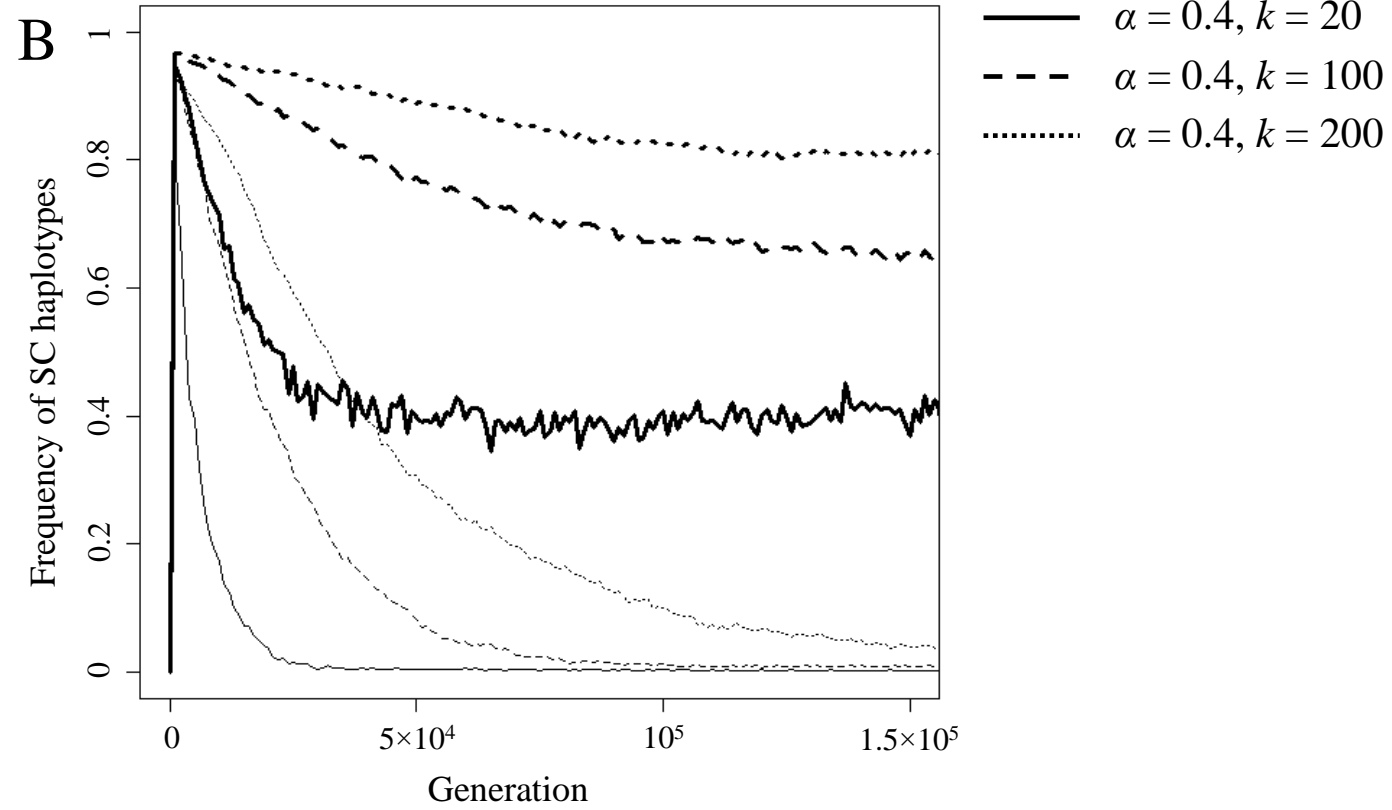

Fig. S3: Dynamics of the number of haplotypes for different combinations of self-pollination rate $\alpha$ and number of specificities $k$. Thick line: $\alpha=0.2$, thin line: $\alpha=0.4$, continuous line: $k=20$, dashed line: $k=100$, dotted line: $k=200$. Dynamics are averages over 100 replicates and were calculated for $\delta=0.9, N=5,000, p=5$ and $n=5$. A. Number of $S$-haplotypes across $5 \times 10^{5}$ generations. B. Frequency of SC haplotypes across $1.5 \times 10^{5}$ generations. 


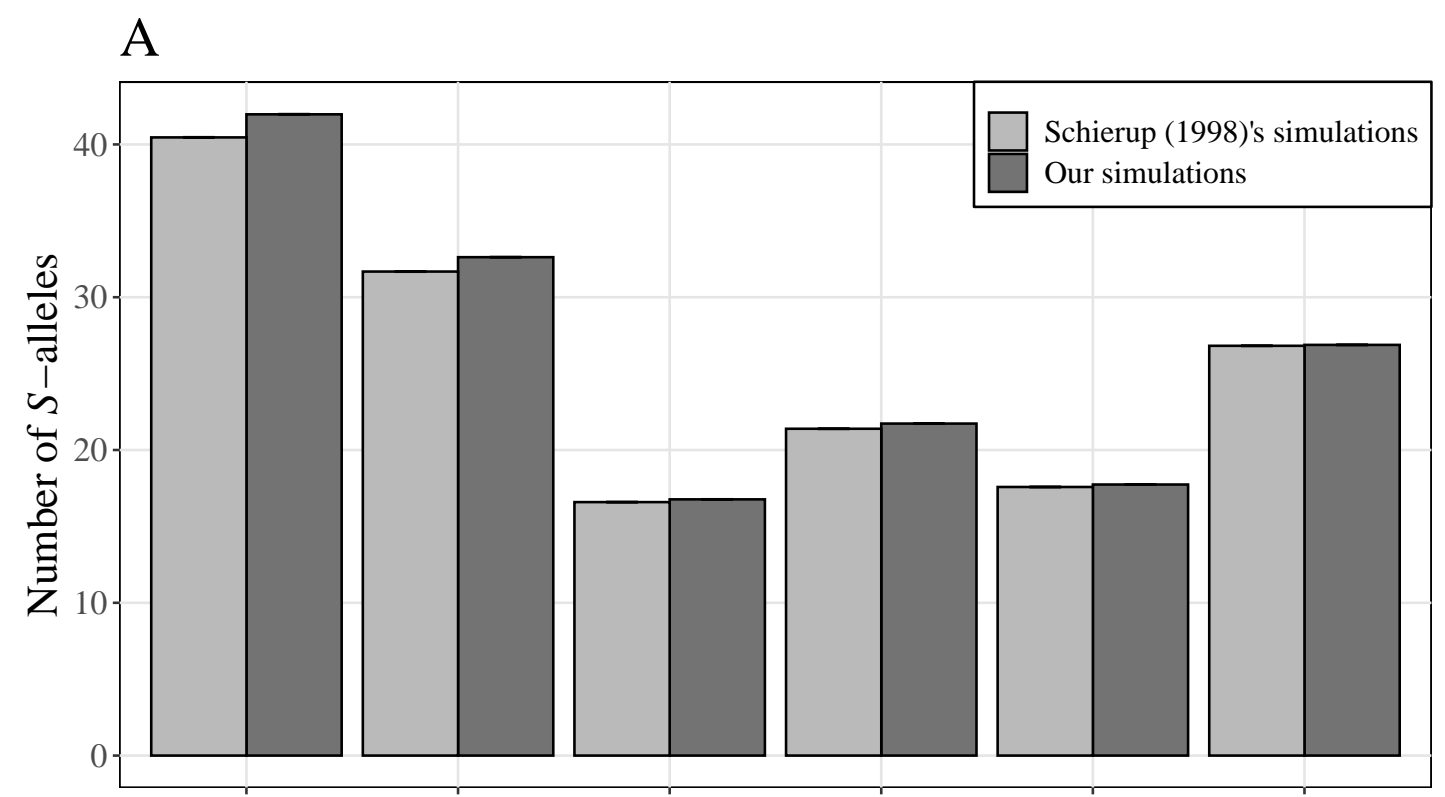

$\mathrm{B}$

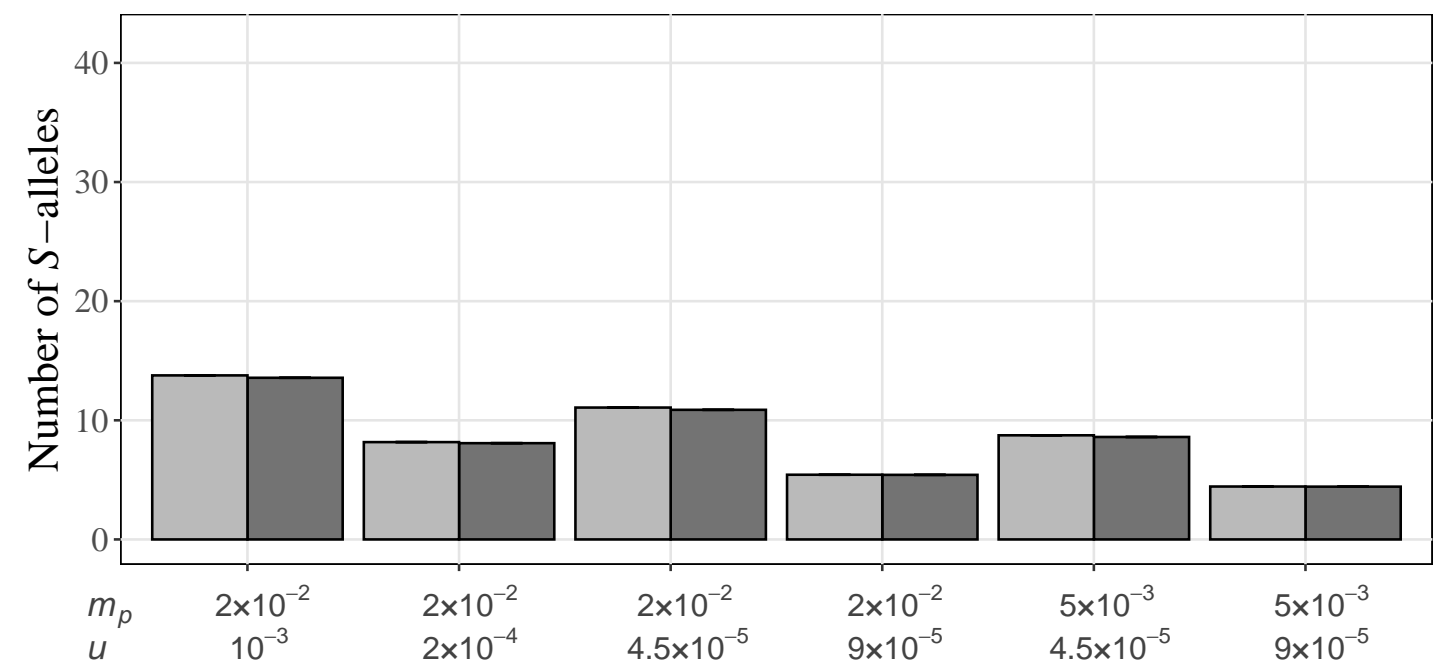

Parameter combination

Fig. S4: Verification that the number of $S$-alleles in the population (A) and average number of alleles per deme (B) calculated with our simulation program (light-grey bars) matches those in Schierup (1998) (dark-grey bars) with the method detailed in Schierup (1998) for different combinations of parameters (pollen migration rate $m_{p}$ or $d_{p}$ and mutation rate $u$ or $\mu_{1}$ ).

Each bar represents the average number of alleles over 100 replicates and the interval $\pm \mathrm{SE}$ is represented by error bars (often as thick as the upper line of the bar). 


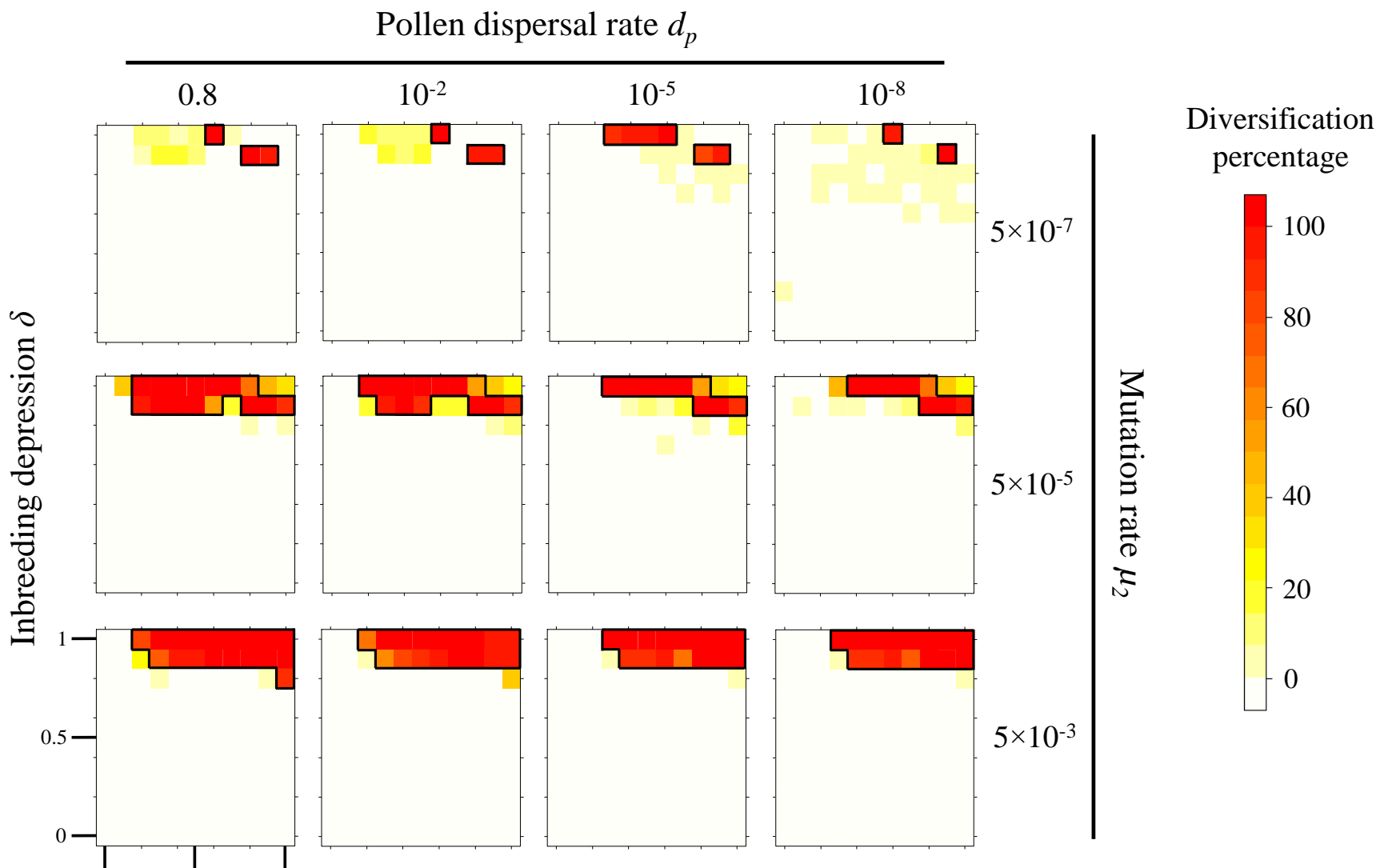

\section{Self-pollination rate $\alpha$}

Fig. S5: Parameter regions allowing diversification of $S$-haplotypes as a function of mutation rate $\mu$ and pollen dispersal rate $d_{p}$. The colour range indicates the diversification percentage (the number of times diversification occurred over 100 replicates) and goes from white, meaning no diversification occurred, to dark red, meaning diversification always occurred. Regions surrounded in black correspond to combination of parameters simulated to study the haplotype number at equilibrium $\widehat{n}$ (Fig. 4). Each grid cell corresponds to 100 replicates. For all simulations $N=5000, p=5, n=5$ and $k=20$. 
A

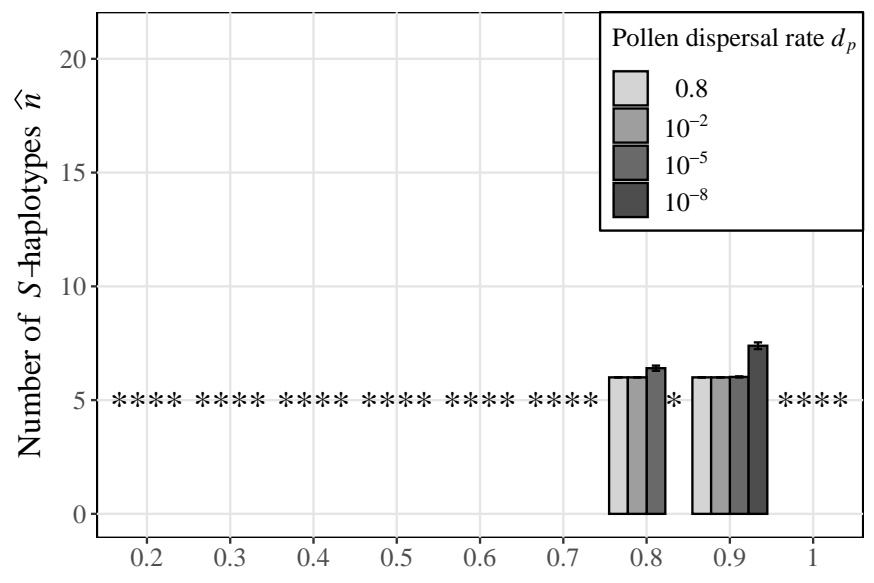

$\mathrm{C}$

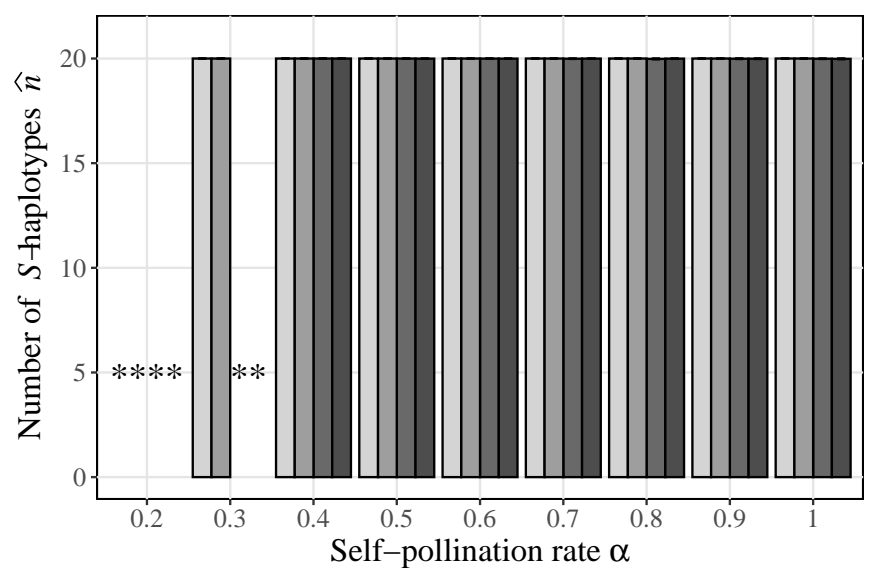

$\mathrm{B}$

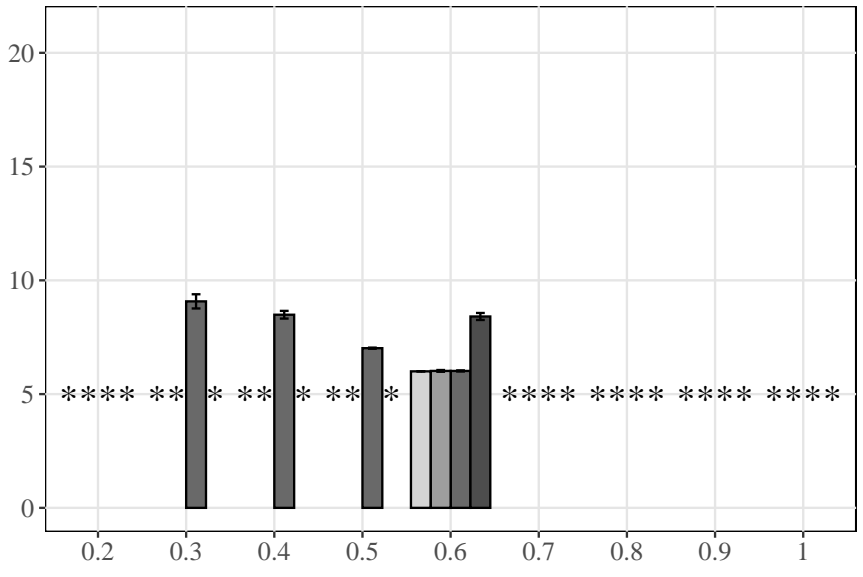

$\mathrm{D}$

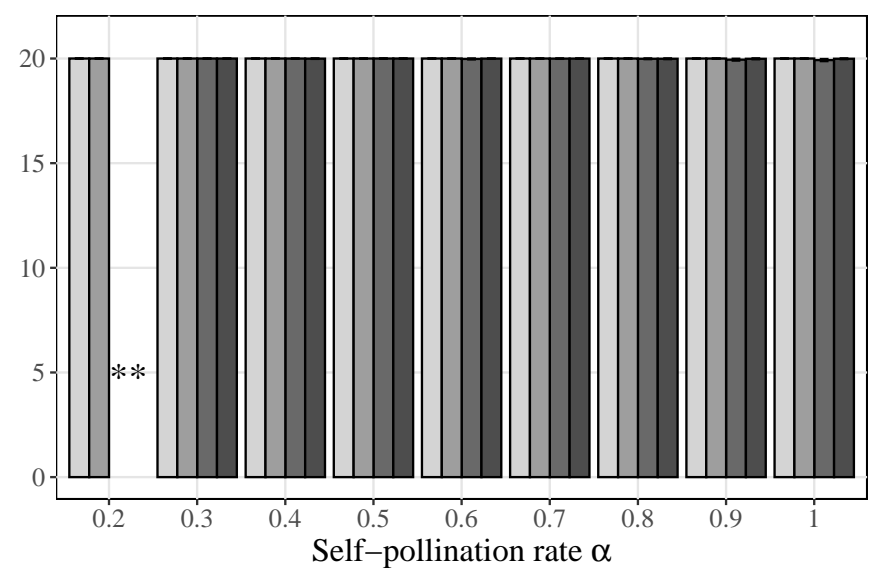

Fig. S6: Average number of $S$-haplotypes at steady state $\widehat{n}$ and $95 \%$ CI (not visible when too small) as a function of the self-pollination rate $\alpha$ and for different pollen dispersal rate $d_{p}$. An asterisk indicates a combination of parameters where diversification is too rare to be considered (see text for details, and see Fig. 3). Parameters: $N=5000, p=5, n=5$ and $k=20$. A: $\delta=0.9$, $\mu=5 \times 10^{-7}$; B: $\delta=1, \mu=5 \times 10^{-7}$; C: $\delta=0.9, \mu=5 \times 10^{-3}$; D: $\delta=1, \mu=5 \times 10^{-3}$. 